\title{
Zeeman Topologies on Space-Times of General Relativity Theory
}

\author{
Rüdiger Göbel \\ Fachbereich 6, Mathematik, Universität Essen, GHS, D-4300 Essen 1, Federal Republic of Germany
}

\begin{abstract}
In 1964 Zeeman published a paper showing [independently of Alexandrov (1953)] that the causal structure of the light cones on Minkowski space $M$ determines the linear structure of $M$. This initiated the question whether a topology (more physically than the ordinary one) on $M$, related to the light cones also implies the linear structure of $M$. In 1967 Zeeman defined such a new topology - here called Zeeman-topology $3_{0}$ - on Minkowski space and solved this question for $M$. In that paper he asked whether it is possible to generalize this program to general relativity. Two of his main questions were: (a) What is the structure of the group $G(S)$ of all homeomorphisms of a spacetime $S$ with respect to the general relativistic analogue of $3_{0}$ (defined in $\S 3$ )? (b) What are the world lines (defined in $\S 1$ ) with respect to $3_{0}$ ? Without any restrictions on the space-time $S$ we will give the answers: (a) $G(S)$ is the group of all homothetic transformations on $S$ (for an explicite discussion of this result we refer to $\S 5$ ). (b) World lines are broken geodesics. Including external fields (like Maxwell fields and deviations of $3_{0}$ ) the answer $(b)$ can be generalized in different physical directions; cf. $\S 3$.
\end{abstract}

Ein Fernrohr wird gezeigt, womit man seinen eigenen Rücken sieht.

Es führt durchs Weltall deinen Blick im Kreis zurück auf dein Genick.

Zwar braucht es so geraume Frist, daß du schon längst verstorben bist, doch wird ein Standbild dir geweiht, empfängt es ihn zu seiner Zeit.

Christian Morgenstern, ,Böhmischer Jahrmarkt“

\section{Contents}

$\S$ 1. Introduction and Discussion . . . . . . . . . . . . . . . . . . . . . . . . . . 290

§2. Definition and Notations . . . . . . . . . . . . . . . . . . . . . . . . . . . 292

§3. Zeeman Topology on a General Relativistic Space-Time . . . . . . . . . . . . . . . . 293

§4. Further Properties of the Zeeman Topologies on Space-Times . . . . . . . . . . . . . . . 297

§ 5. The Group of All Homeomorphisms of a Space-Time . . . . . . . . . . . . . . . . . 298

$\S 6$. References . . . . . . . . . . . . . . . . . . . . . . . . . . . . . . . . . . 306 


\section{$\S 1$. Introduction and Discussion}

The aim of this paper is to answer two questions of Zeeman [38, p. 162, first paragraph and problem (1)]. Zeeman [38] introduced a new topology ${ }^{1} 3_{0}$ $\left[3_{0}=3\left(\Sigma_{0}, \mathfrak{T}\right) ; \mathrm{cf} . \S 3(*)\right.$, and $\S 4(\mathrm{I})$ and $\left.\left(\mathrm{II}_{0}\right)\right]$ on Minkowski space. This topology $3_{0}$ has many physically attractive properties. In particular 30 is defined in a much more intuitive and physical way than the ordinary Euclidean topology $\mathfrak{I}$ which is artificial from both the mathematical as well as the physical point of view. The topology $\mathfrak{I}$ (on a Minkowski space) can be understood primarily from its history: People were mostly concerned with Riemannian spaces (with a positive metric) for which $\mathfrak{I}$ is a very natural topology, and not with spaces provided with a pseudo-Euclidean metric or in particular with a Lorentz metric. The latter have been ignored in mathematics for a long time; cf. Freudenthal [11, p. 18]. As suggested by Zeeman [38, p. 162, (1)] we will extend his topology to arbitrary space-times of general relativity theory. At the same time it is possible to generalize his results on special relativity to general relativity theory without any restrictions (Corollary 3.6 and Corollary 5.11).

The characterizing property of the (ordinary Euclidean) manifold topology $\mathfrak{I}$ on a space-time "to be locally Euclidean" is not plausible and can hardly be justified. In particular there are no experiments known to justify a Euclidean topology along light like geodesic lines, which are the world lines of photons. On the other hand the (induced) topology along the track of a photon is Euclidean in consequence of the manifold topology $\mathfrak{I}$. Because of the light cone at a point and the local future and past at a point, the space-time is locally not "spherically symmetric". The topology $\mathfrak{I}$ does not reflect this very important feature of spacetimes, on the contrary, $\mathfrak{I}$ is invariant under arbitrary 4-dimensional "rotations". These objections to $\mathfrak{I}$ and the fact that local time distances of an observer and local particle distances in space like hypersurfaces are physically plausible lead to the definition of the new topology $3_{0}$ on a space-time: Generalizing Zeeman [38] we define the Zeeman topology $\mathbf{Z}_{0}$ as the finest topology on a space-time such that its induced topology on world lines of freely falling test particles with positive rest mass, and on space like hypersurfaces is locally Euclidean (cf. $\$ \$ 3$ and 4).

Zeeman topology is (by definition) not as "nice" as the manifold topology, e.g. it is not "normal" in the sense of topology; cf. Zeeman [38, p. 164, Remark] but it has many physically interesting properties:

The fact that we do not have any geometric information along a light ray, can be observed of the Zeeman topology: (Corollary 4.2) The topology induced by the Zeeman topology on a light cone is discrete (which means there is no topological "information" on light rays). A world line is the continuous image $(f:[\mathbf{0}, \mathbf{1}] \rightarrow \boldsymbol{M})$ of the unit interval in the space-time $\boldsymbol{M}$ such that the defining map $f$ preserves the natural order on $[\mathbf{0}, \mathbf{1}]$ and the locally causal order of future-past ("«", cf. §2) in the space-time. There are many unphysical world lines, e.g. "bad trips" in the sense of Penrose [25, p. 3]. Only under further restrictions we obtain a physically realistic notation of "world line of a test particle". If however we interpret continuity of $f$ with respect to Zeeman topology, world lines are auto-

Further topologies on Minkowski space may be found in Cel'nik [7, p. 1151] and Zeeman [38, pp. 169, 170]. 
matically physically realistic, namely piecewise geodesics which are future directed and time like with finitely many edges (Remark of Corollary 3.6). This was conjectured by Zeeman. Therefore a world line is the orbit of a freely falling test particle within the gravitational field with a finite number of collisions. This result is the well known basic assumption for a kinetic theory in general relativity theory; cf. Ehlers [9, p. 29]. It is only natural to ask whether it is possible to incorporate the electro-magnetic fields into this result. If we allow the Zeeman topology to depend on a gravitational field as well as on the Maxwell field it is in fact possible to derive the corresponding result for charged particles (cf. Corollary 3.6).

The group of all homeomorphisms of a space-time with its manifold topology $\mathfrak{I}$ is neither of interest for physics nor for mathematics since it is vast and seems to reflect no information of the space-time. The situation is completely different if we understand homeomorphisms to be defined with respect to the Zeeman topology (Corollary 5.11): The group $\operatorname{Hom}\left(\boldsymbol{M}, \mathbf{3}_{0}\right)$ of all homeomorphisms of a space-time $\boldsymbol{M}$ with respect to its Zeeman topology $3_{0}$ coincides with its group $\operatorname{Hot}(\boldsymbol{M})$ of all homothetic transformations (cf. $\S 2$ ), i.e. homeomorphisms are isometries or isometries up to a constant positive factor. Therefore homeomorphisms are proper symmetry transformations of the space-time. If $\boldsymbol{M}$ is the Minkowski space, the homothetic transformations are just the Lorentz transformations or dilatations of Minkowski space. Therefore the homeomorphism group $\operatorname{Hom}\left(\boldsymbol{M}, \boldsymbol{3}_{0}\right)$ of Minkowski space is its Weyl group, which is generated by Lorentz transformations and (linear) dilatations. (It might be interesting to recall that there are many topological groups which can never be represented as full homeomorphism groups with the compact-open topology of some topological space; cf. Brechner [6].) Our result (Corollary 5.11), that the symmetry group and in particular the Lorentz group can be derived from continuity of the maps only, belongs to a quite popular problem which has been investigated very often from many points of view: Is it possible to derive a particular group of (differentiable/linear) isometries from appearently much weaker conditions without any further assumptions (like differentiability, linearity, ...) on the maps? Typical (weaker symmetry) conditions are: inertial frame preserving, metric preserving, causality preserving, order preserving, cone preserving, geodesic preserving or topology preserving. Corresponding investigations may be found in Vock [32, pp. 15-22], Nevanlinna [24, pp. 159-177], Süßmann [29], Loewner [21], Weyl [35], Rätz [26], Knichal [39], Pimenov [40], Vogt [33], Hawking [15], Michel [22], Göbel [13], Benz [4], Barucchi [3], Alfonso and Ynduráin [2], Borchers and Hegerfeldt [5, 17], Gheorghe and Mihul [12], Guts [14], Teppati [30], Zeeman [37], Alexandrow [1, 41], Rothaus [27], Vroegindewey [34], Vilms [31] and Zeeman [38]; see also Domiaty [8].

Another way of looking at our main result (Theorem 5.9) is as follows (Corollary 5.10): Two space-times are homeomorphic with respect to its Zeeman topologies if and only if they are isometric. Therefore it is possible to determine the metric of a space-time from its Zeeman topology 3. . A result like this might be useful for reconstructing a space-time of general relativity on an axiomatic way; cf. Ehlers and Schild [10].

Furthermore I would like to remark that there are some other physically plausible topologies suggested by Zeeman [38, pp. 169, 170] and by Hawking 
(oral communication, cf. $\S 3$ ) which can be transfered to general relativity. It is to be expected that the results for these topologies are very similar to that for $3{ }_{0}{ }^{2}$

In our proofs we will follow the ideas of Zeeman's [38] and apply results of Hawking, Penrose, and Göbel [13]. We will start off with a very general definition of "Zeeman topologies" [which includes all other suggested topologies on spacetimes; cf. $\S 3(*)$ ] so that it will be possible to derive results for arbitrary manifolds as well; cf. Corollary 3.5. The general setting of "Zeeman topologies" will be restricted more and more, and at the end (\$5) we will specialize it for $3_{0}$ only.

I would like to express my gratitude in particular to Rudolf Z. Domiaty, Jürgen Ehlers, Steven W. Hawking, Christopher Zeeman and my former colleagues Werner Dietz and Roland Rüdiger for stimulating discussions on this subject.

\section{§ 2. Definition and Notations}

Let $\boldsymbol{X} \cong \boldsymbol{Y}$, i.e. $\boldsymbol{X}$ is a subset of $\boldsymbol{Y}$, then $\boldsymbol{Y} \backslash \boldsymbol{X}=\{x \in \boldsymbol{Y}$ such that $x \notin \boldsymbol{X}\}$ is the complement of $\boldsymbol{X}$ in $\boldsymbol{Y}$. Let $\boldsymbol{M}=(\boldsymbol{M}, \mathfrak{I})$ be a topological space with topology $\mathfrak{I}=\mathfrak{I}(\boldsymbol{M})$ consisting of all (with respect to $\mathfrak{I}$ ) open subsets of $\boldsymbol{M}$. If $\boldsymbol{U} \cong \boldsymbol{M}$, then $\mathfrak{I}_{\boldsymbol{U}}=\{\boldsymbol{X} \cap \boldsymbol{U} ; \boldsymbol{X} \in \mathfrak{I}(\boldsymbol{M})\}$ defines the induced topology on $\boldsymbol{U}$, and $\left(\boldsymbol{U}, \mathfrak{I}_{\boldsymbol{U}}\right)$ is a topological space of its own.

A space-time in general relativity theory is a 4-dimensional, time oriented, connected $\mathscr{C}^{d}$-manifold $(d=$ degree of differentiability $>2)$ with a $\mathscr{C}^{d-1}$-Lorentz metric $\boldsymbol{g}=\boldsymbol{g}($,$) ; cf. Ehlers [9, p. 3] and Hawking and Ellis [16, p. 56].$

If $\boldsymbol{M}$ is a space-time, $\boldsymbol{U} \subseteq \boldsymbol{M}$ and $P, Q \in \boldsymbol{M}$, then we have $P \ll_{\boldsymbol{U}} Q$ (say: the event $P$ is in the past of the event $Q$ within $\boldsymbol{U}$ ), if there is a differentiable, time like map $f:[\mathbf{0 , 1}] \rightarrow \boldsymbol{U}$ connecting $P=f(0)$ with $Q=f(1)$ such that all tangents along $f$ are future directed. This means physically that there is a possibility to send a test particle of positive rest mass from the event $P$ to the event $Q$ within the region $\boldsymbol{U}$. If $P \in \boldsymbol{U} \cong \boldsymbol{M}$, we denote by $\mathfrak{I}_{\boldsymbol{U}}^{+}(P)$ the set of all $Q \in \boldsymbol{U}$ such that $P \ll_{\boldsymbol{U}} Q$, and similar by $\mathfrak{I}_{\boldsymbol{U}}^{-}(P)$ the set of all $Q \in \boldsymbol{U}$ such that $Q \ll_{\boldsymbol{U}} P$. Then $\mathfrak{I}_{\boldsymbol{U}}^{+}(P)\left[\mathfrak{I}_{\boldsymbol{U}}^{-}(P)\right]$ is called the chronological future [past] of $P$ relative to $\boldsymbol{U}$; cf. Hawking and Ellis [16, p. 182]. Furthermore we denote by $\Xi_{v}(P)$ the set of all $Q \in U$ such that there is a differentiable map $f:[\mathbf{0}, \mathbf{1}] \rightarrow \boldsymbol{U}$ whose tangents are space like and with $f(0)=P$ and $f(1)=Q$. We call $\mathfrak{S}_{\boldsymbol{U}}(P)$ the space like part of $P$ relative to $\boldsymbol{U}$. Similarly, let $\mathfrak{C}_{\boldsymbol{U}}^{+}(P)\left[\mathfrak{C}_{\boldsymbol{U}}^{-}(P)\right]$ be the future [past] light cone of $P$ relative to $\boldsymbol{U}$ where the tangents in consideration are null vectors and future [past] directed; let be

$$
\mathfrak{C}_{\boldsymbol{U}}(P)=\mathfrak{C}_{\boldsymbol{U}}^{+}(P) \cup \mathfrak{C}_{\boldsymbol{U}}^{-}(P)
$$

the light cone at $P$ relative to $\boldsymbol{U}$. We assume $P \notin \mathfrak{I}_{\boldsymbol{U}}^{ \pm}(P)$ but $P \in \mathfrak{C}_{\boldsymbol{U}}^{ \pm}(P)$ and $P \in \mathfrak{S}_{\boldsymbol{U}}(P)$ ! It is conventional to drop the index $\boldsymbol{U}$ if $\boldsymbol{U}=\boldsymbol{M}$.

A subset $\boldsymbol{S}$ of a space-time $\boldsymbol{M}$ is called simple region, if $\boldsymbol{S}$ has the following properties:

(a) $\boldsymbol{S}$ is an open subset of $\boldsymbol{M}$ (with respect to the manifold topology).

(b) If $P, Q \in \boldsymbol{S}$ there is one and only one $P$ with $Q$ connecting geodesic curve which we denote by $\overline{P Q}$. The geodesic $\overline{P Q}$ lies entirely in $S$.

(c) Each geodesic curve $\overline{P Q}$ defined by (b) which belongs to $S$ depends continuously on $P$ and $Q$.

(d) The boundary $\partial \boldsymbol{S}$ of $\boldsymbol{S}$ and all closed subsets of $\boldsymbol{S}$ are compact.

2 Note Added in Proof. These topologies will be considered in two forthcoming papers by Hawking, King, McCarthy [42] and Göbel [43]. In [43] the results of [23] will be corrected. 
It was shown by Penrose [25, p. 6, Proposition 1.13] that any space-time can be covered by a locally finite system of simple regions. We will make extensive use of this fact. In particular a space-time $\boldsymbol{M}$ is locally causal; cf. Penrose [25, Proposition 4.12]. If $\overline{P Q}$ is a geodesic line - defined as above by $(\mathrm{b})-$ we write $(\overline{P Q})=$ $\overline{P Q} \backslash\{P, Q\}$ to indicate that the end points are taken away.

A map $f$ from a space-time $\boldsymbol{M}$ into a space-time $\boldsymbol{L}$ is called locally causal, if for each point $P \in \boldsymbol{M}$ there is a simple region $\boldsymbol{U}$ of $\boldsymbol{M}$ containing $P$, and a simple region $\boldsymbol{V}$ of $\boldsymbol{L}$ containing $f(P)$ with the following properties:

(1) We have $f(\boldsymbol{U}) \subseteq \boldsymbol{V}$.

(2) For each pair $X, Y \in \boldsymbol{U}$ we have $X \ll_{\boldsymbol{U}} Y$ or $Y \ll_{\boldsymbol{U}} X$ if and only if $f(X) \ll_{V} f(Y)$ or $f^{\prime}(Y) \ll_{V} f(X)$.

If $f^{\prime}$ is a $1-1$-map of $\boldsymbol{M}$ onto $\boldsymbol{L}$ such that $f$ and $f^{-1}$ are locally causal, the spaces $\boldsymbol{L}$ and $\boldsymbol{M}$ are locally causally equal. A homeomorphism of $(\boldsymbol{M}, \mathfrak{I}(\boldsymbol{M}))$ onto $(\boldsymbol{L}, \mathfrak{I}(\boldsymbol{L}))$ is a $1-1$-map of $\boldsymbol{M}$ onto $\boldsymbol{L}$, which maps $\mathfrak{I}(\boldsymbol{M})$ onto $\mathfrak{I}(\boldsymbol{L})$. To indicate the underlying topologies we sometimes call this map a $\mathfrak{I}$-homeomorphism. The spaces $\boldsymbol{L}$ and $\boldsymbol{M}$ are homeomorphic. If $f$ is a diffeomorphism from $\boldsymbol{M}$ onto $\boldsymbol{L}$, and $f_{*}$ is the induced map within the corresponding tangent spaces of $\boldsymbol{M}$ and $\boldsymbol{L}$, the map $f$ is called conformal map of $\boldsymbol{M}$ onto $\boldsymbol{L}$ with conformal "factor" $\Omega$ if $\Omega$ is a nowhere vanishing, differentiable map from $\boldsymbol{M}$ into the real numbers with the following property:

$$
\boldsymbol{g}_{f(X)}\left(f_{*} \boldsymbol{t}_{X}, f_{*} \boldsymbol{s}_{X}\right)=\Omega(X)^{2} \cdot \boldsymbol{g}_{X}\left(\boldsymbol{t}_{X}, \boldsymbol{s}_{X}\right)
$$

for all tangents $\boldsymbol{t}_{X}, \boldsymbol{s}_{X}$ at all points $X \in \boldsymbol{M}$. $\left[\boldsymbol{g}_{X}(\right.$,$) is the metric of \boldsymbol{M}$ at $X$ and $\boldsymbol{g}_{f(X)}($,$) is the metric attached at f(X)$ of $\boldsymbol{L}$.] The spaces $\boldsymbol{L}$ and $\boldsymbol{M}$ are called conformally equal. If $\Omega$ is a constant, $f$ is called a homothetic transformation of $\boldsymbol{M}$ onto $\boldsymbol{L}$; cf. e.g. Kobayashi and Nomizu [20, p. 309]. If $\Omega=1, f$ is called an isometry, and $\boldsymbol{L}$ and $\boldsymbol{M}$ are isometric.

\section{$\S 3$. Zeeman Topology on a General Relativistic Space-Time}

At the moment we reserve $\boldsymbol{M}=(\boldsymbol{M}, \mathfrak{I})$ to denote a differentiable manifold with an underlying manifold topology $\mathfrak{I}=\mathfrak{T}(\boldsymbol{M})$. Later on it will be a space-time for general relativity theory. If $f:[\mathbf{0}, \mathbf{1}] \rightarrow \boldsymbol{M}$, we denote by $\boldsymbol{f}=f[\mathbf{0}, \mathbf{1}]$ the image point set with the induced topology $\mathfrak{I}_{\boldsymbol{f}}$. We say $\boldsymbol{f}$ is a curve if $f$ is a continuous $1-1$-map, and $f$ is a differentiable curve if $f$ is in addition differentiable.

The most general setting for Zeeman topologies ${ }^{3}$ is the following: Let $\Sigma$ be a set of subsets of $\boldsymbol{M}$.

(*) Then a subset $\boldsymbol{X} \cong \boldsymbol{M}$ belongs to $\boldsymbol{Z}=3(\Sigma, \mathfrak{I})$ if and only if $\boldsymbol{X} \cap \boldsymbol{Y}$ is open within the topological space $\boldsymbol{Y}=\left(\boldsymbol{Y}, \mathfrak{I}_{\boldsymbol{Y}}\right)$ with its induced topology $\mathfrak{I}_{\boldsymbol{Y}}$ for all $\boldsymbol{Y} \in \Sigma$.

Then $(\boldsymbol{M}, \mathbf{3})$ is the space $\boldsymbol{M}$ provided with the Zeeman topology $3=3(\Sigma, \mathfrak{I})$ generated by $(\Sigma, \mathfrak{I})$.

The topology 3 is the finest topology $\mathfrak{F}$ on $\boldsymbol{M}$ such that $\mathfrak{F}_{\boldsymbol{Y}}=\mathfrak{I}_{\boldsymbol{Y}}$ for all $\boldsymbol{Y} \in \Sigma$. Zeeman [38] introduced this type of topology on Minkowski space for two specially chosen systems $\Sigma$ which are significant for special relativity. Since a $\mathfrak{I}$-open subset of $\boldsymbol{M}$ satisfies (*), the Zeeman topology is always finer than $\mathfrak{I}$.

3 Cf. Cel'nik [7.p. 1151]. 
There are two exceptional cases of Zeeman topologies if $\Sigma=\emptyset$ and if $\Sigma=\{\boldsymbol{M}\}$. In the first case the Zeeman topology is the discrete topology on $\boldsymbol{M}$ and in the second case it is the manifold topology $\mathfrak{I}$. In general we have $\mathfrak{I}<3$ and there are

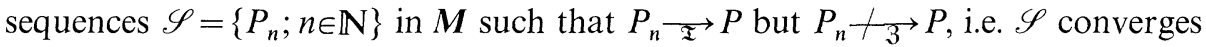
to $P$ with respect to $\mathfrak{I}$ but not with respect to 3 . Such sequences are called Zeno sequences; cf. Zeeman [38, p. 164]. They may be constructed as follows:

Lemma 3.1. Let $\Sigma$ be a set of closed subsets of $\boldsymbol{M}$. For a sequence $\mathscr{S}$ of points $\neq P$ of $\boldsymbol{M}$ which converges to $P$ with respect to $\mathfrak{I}$ are equivalent:

(a) $\boldsymbol{U}^{*}=\boldsymbol{U} \backslash \mathscr{S}$ is open in the Zeeman topology $3=3(\Sigma, \mathfrak{I})$ for all $\boldsymbol{U} \in \mathfrak{I}$.

(b) If $P \in \boldsymbol{X} \in \Sigma$ then $|\boldsymbol{X} \cap \mathscr{P}|<\infty$.

In particular $\mathscr{S}$ is a Zeno sequence.

Proof. (a) $\rightarrow$ (b): Let $\mathscr{S}^{\prime}=\boldsymbol{X} \cap \mathscr{S}$ be infinite for some $P \in \boldsymbol{X} \in \Sigma$. Then $P$ is an accumulation point of $\mathscr{S}^{\prime}$ in $\left(\boldsymbol{X}, \mathfrak{I}_{\mathbf{X}}\right)$, but $P \notin \mathscr{S}^{\prime}$. Therefore $\mathscr{S}^{\prime}$ is not $\mathfrak{I}_{\boldsymbol{X}}$-closed, and $\boldsymbol{U}^{*} \cap \boldsymbol{X}=(\boldsymbol{U} \backslash \mathscr{S}) \cap \boldsymbol{X}=(\boldsymbol{U} \cap \boldsymbol{X}) \backslash(\boldsymbol{X} \cap \mathscr{S})=(\boldsymbol{U} \cap \boldsymbol{X}) \backslash \mathscr{S}^{\prime}$ is not open in $\mathfrak{T}_{\boldsymbol{X}}$ for all $\boldsymbol{U} \in \mathfrak{I}$ with $P \in \boldsymbol{U}$. In particular $\boldsymbol{U}^{*}$ is not 3 -open. (b) $\rightarrow(\mathrm{a})$ : We assume $\mathscr{S}^{\prime}=\boldsymbol{X} \cap \mathscr{S}$ to be infinite for some $\boldsymbol{X} \in \Sigma$. Then $\mathscr{S}^{\prime}$ is a partial sequence of $\mathscr{S}$ contained in $\boldsymbol{X}$ which converges to $P$ with respect to $\mathfrak{I}$. Therefore $P \in \boldsymbol{X}$, since $\boldsymbol{X}$ is $\mathfrak{I}$-closed, which contradicts (b). Therefore we have

(c) $|X \cap \mathscr{S}|<\infty$ for all $\boldsymbol{X} \in \Sigma$.

The topology $\mathfrak{I}$ is Hausdorff, and therefore a finite point set, and in particular $\mathscr{S} \cap \boldsymbol{X}$, is closed for all $\boldsymbol{X} \in \Sigma$ by (c). If $\boldsymbol{U} \in \mathfrak{I}$ we have $\boldsymbol{U} \cap \boldsymbol{X}$ open in $\left(\boldsymbol{X}, \mathfrak{I}_{\boldsymbol{X}}\right)$, hence $\boldsymbol{U}^{*} \cap \boldsymbol{X}=(\boldsymbol{U} \backslash \mathscr{S}) \cap \boldsymbol{X}=(\boldsymbol{U} \cap \boldsymbol{X}) \backslash(\mathscr{S} \cap \boldsymbol{X})$ is open in $\left(\boldsymbol{X}, \mathfrak{I}_{\boldsymbol{X}}\right)$ for all $\boldsymbol{X} \in \Sigma$. Therefore $\boldsymbol{U}^{*}$ is open with respect to the Zeeman topology. If we choose $P \in \boldsymbol{U} \in \mathfrak{T}$, the set $\boldsymbol{U}^{*}=\boldsymbol{U} \backslash \mathscr{S}$ is a 3 -neighbourhood of $P$, and $P_{n}{ }_{3} P$ follows.

Since we are not interested in the general situation of this topology 3 , we are going to make restrictions on $\Sigma$. The restrictions are chosen in such a way, that it is possible to consider (certain classes of) Zeeman topologies on Riemannian manifolds as well as on arbitrary space-times. Under these restrictions we can construct Zeno sequences according to Lemma 3.1.

$(* *)$ We call $\Sigma=(\Gamma, \Delta)$ a special system of $\boldsymbol{M}$, if there is a locally finite covering $\mathfrak{U}$ of $M$ by neighbourhoods $\boldsymbol{U}$, such that $\Gamma=\bigcup_{\boldsymbol{U} \in \mathfrak{U}} \Gamma_{\boldsymbol{U}}$ and $\Delta=\bigcup_{\boldsymbol{U} \in \mathfrak{U}} \Delta_{\boldsymbol{U}}$ where $\Gamma_{\boldsymbol{U}}=\{\boldsymbol{X} \in \Gamma, \boldsymbol{X} \subseteq \boldsymbol{U}\}$ and $\Delta_{\boldsymbol{U}}=\{\boldsymbol{X} \in \Delta, \boldsymbol{X} \subseteq \boldsymbol{U}\}$ have the following properties:

(i) If $\boldsymbol{X} \in \Sigma=\Gamma \cup \Delta$, then $\boldsymbol{X}$ is a closed subset of $\boldsymbol{M}$.

(ii) If $\boldsymbol{X} \in \Gamma_{\boldsymbol{U}}, \boldsymbol{Y} \in \Gamma_{\boldsymbol{V}}$ and $|\boldsymbol{X} \cap \boldsymbol{Y}|=\infty$, then $\boldsymbol{X} \cap \boldsymbol{V}=\boldsymbol{Y} \cap \boldsymbol{U}$ for all $\boldsymbol{U}, \boldsymbol{V} \in \mathfrak{U}$.

(iii) If $P, Q \in \boldsymbol{U} \in \mathfrak{U}$ and $\Gamma_{\boldsymbol{U}}(P, Q)=\left\{\boldsymbol{X} \in \Gamma_{\boldsymbol{U}}, P, Q \in \boldsymbol{X}\right\}$ is infinite, then $P=Q$.

(iv) We have $|\boldsymbol{X} \cap \boldsymbol{Y}| \leqq 1$ for all $\boldsymbol{X} \in \Gamma$ and $\boldsymbol{Y} \in \Delta$.

Lemma 3.2. Let $\Sigma$ be a special system and $\mathscr{S}=\left\{P_{n} ; n \in \mathbb{N}\right\}$ be a sequence of points of $\boldsymbol{M}$ with the following properties:

(a) $P_{n} \longrightarrow P$, but $P \neq P_{n} \in \boldsymbol{U} \in \mathfrak{U}$ (defined by (**)) for all $n \in \mathbb{N}$.

(b) $\Gamma_{\boldsymbol{V}}\left(P, P_{n}\right) \cap \Gamma_{\boldsymbol{V}}\left(P, P_{m}\right)=\emptyset$ for all $n, m \in \mathbb{N}, n \neq m$ and $\boldsymbol{V} \in \mathfrak{H}$.

(c) $\Gamma_{\boldsymbol{U}}\left(P, P_{n}\right) \neq \emptyset$ for all $n \in \mathbb{N}$.

Then $\mathscr{S}$ is a Zeno sequence and $\boldsymbol{W}^{*}=\boldsymbol{W} \backslash \mathscr{S}$ is a $3(\Sigma, \mathfrak{I})$-neighbourhood for all $\boldsymbol{W} \in \mathfrak{T}$.

Proof. We assume $|\mathscr{S} \cap \boldsymbol{X}|=\infty$ for some $P \in \boldsymbol{X} \in \Sigma$. If $\boldsymbol{X} \in \Gamma_{\boldsymbol{V}}$, we get $P, P_{n} \in \boldsymbol{X}$ for infinitely many $P_{n} \in \mathscr{S}$, and we obtain $X \in \Gamma_{\boldsymbol{V}}\left(P, P_{n}\right) \cap \Gamma_{\boldsymbol{V}}\left(P, P_{m}\right) \neq \emptyset$ for infinitely 
many different points $P_{n}, P_{m} \in \mathscr{S}$, which contradicts (b). If $\boldsymbol{X} \in \Delta_{\boldsymbol{V}}$, then $P_{n}, P \in \boldsymbol{X} \cap \boldsymbol{Y}$, i.e. $|\boldsymbol{X} \cap \boldsymbol{Y}|>1$ for each $\boldsymbol{Y} \in \Gamma_{\boldsymbol{U}}\left(P, P_{n}\right)$ and $P_{n} \in \mathscr{S} \cap \boldsymbol{X}$. Therefore $\Gamma_{\boldsymbol{U}}\left(P, P_{n}\right)=\emptyset$ by (**) (iv), which contradicts (c). We have $|\mathscr{S} \cap \boldsymbol{X}|<\infty$ for all $P \in \boldsymbol{X} \in \Sigma$, and $\boldsymbol{W}^{*}=\boldsymbol{W} \backslash \mathscr{S}$ is a 3 -open set for all $\boldsymbol{W} \in \mathfrak{I}$ by Lemma 3.1. In particular $\mathscr{S}$ is a Zeno sequence of $\boldsymbol{M}$.

A curve $\boldsymbol{f}$ is $\Gamma$-directed at $P \in \boldsymbol{f}$, if there is a neighbourhood $\boldsymbol{U}$ of $P$ defined by (**) such that from $P \neq Q \in \boldsymbol{U} \cap \boldsymbol{f}$ follows $\Gamma_{\boldsymbol{U}}(P, Q) \neq \emptyset$. Furthermore $f:[\mathbf{0}, \mathbf{1}] \rightarrow \boldsymbol{M}$ is a piecewise $\Gamma$-curve at $P=f(a)$, if there are $0 \leqq b<a<c \leqq 1$ such that $f[b, a] \leqq X$ and $f[a, c] \subseteq \boldsymbol{Y}$ for some $\boldsymbol{X}, \boldsymbol{Y} \in \Gamma_{\boldsymbol{U}}$. [If $a=0$ put $b=0$ and if $a=1$ put $c=1$.]

Lemma 3.3. Let $\Sigma$ be a special system of $\boldsymbol{M}$ and $f:[\mathbf{0}, \mathbf{1}] \rightarrow \boldsymbol{M}$ be a $1-1-$ map which is $\Gamma$-directed at $P \in f$. Then $f$ is a piecewise $\Gamma$-curve at $P$ if $f$ is continuous at $P$ with respect to the Zeeman topology $3=3(\Sigma, \mathfrak{I})$.

Proof. We put w.l.g. $P=f(0)$ and assume $f$ not to be a piecewise $\Gamma$-curve at $P$. Since $f:[0, \mathbf{1}] \rightarrow M$ is continuous with respect to 3 at $P$ and $\mathfrak{I} \leqq \mathcal{Z}, f$ is continuous with respect to $\mathfrak{I}$ at $P$. Therefore there is an $a \in(\mathbf{0 ,} \mathbf{1})$ such that $f[0, a]$ is contained in a neighbourhood $\boldsymbol{U}$ of the covering $\mathfrak{U}$ defined by (**). Next we construct a sequence $\mathscr{R}$ of reals $a_{n} \in(0, a)$ such that

(a) $a_{n} \in\left(0, \frac{1}{n}\right)$ for all $n \in \mathbb{N}$.

(b) If $P_{k}=f\left(a_{k}\right)$ then $P_{n} \notin \boldsymbol{X}$ for all $\boldsymbol{X} \in \Gamma_{V}\left(P, P_{i}\right)$, all numbers $i<n$ and all $\boldsymbol{V} \in \mathfrak{U}$ with $P, P_{i} \in V$.

We use complete induction and assume the first $n-1$ points to be defined. If there is no such point $P_{n}=f\left(a_{n}\right)$, this would mean that $f\left[0, \frac{1}{n}\right] \subseteq \bigcup \mathfrak{G}_{n}\left[\right.$ w.l.g. $\left.\frac{1}{n}<a\right]$ where $\mathfrak{G}_{n}=\left\{\boldsymbol{X} ; \boldsymbol{X} \in \Gamma_{\boldsymbol{V}}\left(P, P_{i}\right), P, P_{i} \in \boldsymbol{V} \in \mathfrak{U}, i<n\right\}$.

Since $\mathfrak{U}$ is locally finite and $\left|\Gamma_{\boldsymbol{V}}\left(P, P_{i}\right)\right|<\infty$ by (**) (iii), the set $\left(\mathfrak{b}_{n}\right.$ consists of finitely many closed subsets of $\boldsymbol{M}$. If we now assume that there is no $m \in \mathbb{N}$ and $\boldsymbol{X} \in \mathfrak{G}_{n}$ such that $f\left[0, \frac{1}{m}\right] \leqq \boldsymbol{X}$, there are at least two different $\boldsymbol{X}, \boldsymbol{Y} \in \mathfrak{G}_{n}$ with $|\boldsymbol{X} \cap \boldsymbol{Y}|=\infty$, since $\boldsymbol{G}_{n}$ is finite, the elements of $\boldsymbol{F}_{n}$ are closed in $\boldsymbol{M}$, and $\boldsymbol{f}$ is a homeomorphic image of $[\mathbf{0}, \mathbf{1}]$. This is however, excluded by (**) (ii). Therefore $f\left[0, \frac{1}{m}\right] \subseteq \boldsymbol{X}$ for some $\boldsymbol{X} \in \mathfrak{G}_{n} \subseteq \Gamma, m \in \mathbb{N}$, which is again excluded by our assumption on $\boldsymbol{f}$. Therefore $\mathscr{R}$ can be constructed. Since $\boldsymbol{f}$ is $\Gamma$-directed at $P$ we derive from (a) and (b) the existence of a sequence $\mathscr{S}$ satisfying the suppositions of Lemma 3.2 such that $P_{n}=f\left(a_{n}\right)$ and $a_{n} \rightarrow 0$. Therefore $\mathscr{S}$ is a Zeno sequence by Lemma 3.2, and in particular we have $f\left(a_{n}\right)=P_{n}+3 P=f(0)$. This contradicts the 3 -continuity of $f$ at $P$ and therefore Lemma 3.3 is proved.

A curve $\boldsymbol{f}$ is a piecewise $\Gamma$-curve if there are $0=a_{1}<\ldots<a_{n}=1$ and $\boldsymbol{X}_{i} \in \Gamma$ such that $f\left[a_{i}, a_{i+1}\right] \subseteq \boldsymbol{X}_{i}$ for $i=1, \ldots, n-1$. Since $\boldsymbol{f}$ is compact, we derive from Lemma 3.3 the

Corollary 3.4. Let $\Sigma$ be a special system of $\boldsymbol{M}$ and $f$ be a 3-continuous curve which is $\Gamma$-directed at each point $P \in \boldsymbol{f}$. Then $\boldsymbol{f}$ is a piecewise $\Gamma$-curve. 


\section{Applications of Corollary 3.4}

(A) Let $\mathfrak{U}$ and $\mathfrak{B}$ be two locally finite coverings of a manifold with connection by simple neighbourhoods such that we get: If $V \in \mathfrak{B}$ there is one and only one $\boldsymbol{U}(\boldsymbol{V}) \in \mathfrak{U}$ which contains the closure $\overline{\boldsymbol{V}}$ of $\boldsymbol{V}$, cf. (Kobayashi and Nomizu [20, pp. 166, 167, Theorem 3.6, 3.7]).

If $\boldsymbol{U} \in \mathfrak{U}$, let $\Gamma_{\boldsymbol{U}}$ be the set of all (closed) geodesics in $\overline{\boldsymbol{V}}$ connecting points of the boundary $\partial \overline{\boldsymbol{V}}$ if $\boldsymbol{U}=\boldsymbol{U}(\boldsymbol{V})$ and put $\Delta_{\boldsymbol{U}}=\emptyset$. Then $\Sigma^{0}=\left(\bigcup_{\boldsymbol{U} \in \boldsymbol{U}} \Gamma_{\boldsymbol{U}}, \emptyset\right)$ is a special system of the manifold and we derive from Corollary 3.4 our

Corollary 3.5. For a manifold $(\boldsymbol{M}, \mathfrak{T})$ with an affine connection are equivalent:

(1) The curve $\boldsymbol{f}$ is piecewise geodesic, i.e. $f$ is a broken geodesic line with a finite number of edges.

(2) The 1-1-map $f:[\mathbf{0}, \mathbf{1}] \rightarrow \mathbf{M}$ is continuous with respect to the Zeeman topology $3^{0}=3\left(\Sigma^{0}, \mathfrak{I}\right)$.

(B) Let $\boldsymbol{M}$ be a space-time for general relativity theory and $\mathrm{F}$ a given electromagnetic field on $\boldsymbol{M}$. Particles $\not h$ have to have an electric charge $q_{\not}$ such that its absolute value is bounded by a (sufficiently large) number depending on $\mathrm{F}$ and a mass $m_{\text {h }}>0$, which is bounded by a (sufficiently large) number depending on the gravitational field in order to be test particles. If we take into account the discreteness of charge and mass spectrum, there are finitely many possible values $q_{\hbar} \in \boldsymbol{q}\left(=\right.$ charge spectrum) and $m_{k} \in \boldsymbol{m}$ (=mass spectrum) for test particles $\not h$ only. We assume $0 \in \boldsymbol{q}$, i.e. charge free test particles are always present. If $\boldsymbol{q}=\{0\}$, we allow the mass spectrum $\boldsymbol{m}$ to be arbitrary $>0$. Under these conditions there are coverings $\mathfrak{U}$ and $\mathfrak{B}$ like in (A) so that there are only finitely many world lines of freely falling test particles in $\boldsymbol{U} \in \mathfrak{U}$ from $P \in \boldsymbol{U}$ to $Q \in \boldsymbol{U}$ if $P \neq Q$. (This is a consequence of the Lipshitz condition for the differential equations for the particle orbits.) If $\boldsymbol{U} \in \mathfrak{U}$ let $\Gamma_{q \boldsymbol{U}}^{\boldsymbol{m}}$ be the set of all world lines of freely falling test particles ( $\not$ with $m_{\hat{k}} \in \boldsymbol{m}$ and $\left.q_{h} \in \boldsymbol{q}\right)$ from $P$ to $Q \neq P$ if $P, Q \in \partial \overline{\boldsymbol{V}}$ and $\boldsymbol{U}=\boldsymbol{U}(\boldsymbol{V})$; let $\Delta_{\boldsymbol{U}}$ be all closed space like $\mathscr{C}^{1}$-hypersurfaces of $\overline{\boldsymbol{V}}$. The corresponding system $\Sigma_{\boldsymbol{q}}^{\boldsymbol{m}}$ is a special system of $\boldsymbol{M}$.

World lines are generally defined as curves $\boldsymbol{f}$ such that $f:[\mathbf{0}, \mathbf{1}] \rightarrow \boldsymbol{M}$ is a continuous 1-1-map which preserves the natural order on $[0,1]$ and the locally causal order on $\boldsymbol{M}$; cf. $\S 2$. Then world lines are automatically $\Gamma_{q}^{\boldsymbol{m}}$-directed curves, and we obtain from Corollary 3.4 our

Corollary 3.6. For a space-time $\boldsymbol{M}$ with a given external electro-magnetic field and $a$ world line $f$ are equivalent:

(1) $f$ is continuous with respect to the Zeeman topology $3\left(\Sigma_{q}^{\boldsymbol{m}}, \mathfrak{I}\right)$.

(2) $f$ is a chain of finitely many connected world lines of freely falling charged test particles.

Remark. If no external electro-magnetic field is present [i.e. if $\mathrm{F}=0$ and/or $\boldsymbol{q}=0$ ], we derive from Corollary 3.6 for $\Sigma_{0}^{\boldsymbol{m}}=\Sigma_{0}$ : The $3\left(\Sigma_{0}, \mathfrak{T}\right)$-continuous world lines are future directed, time like piecewise geodesic lines. This answers a conjecture by Zeeman [38, p. 162, first paragraph].

We can say that the gravitational field and the electro-magnetic field determine the topology $3\left(\Sigma_{q}^{m}, \mathfrak{I}\right)$ (like the metric) of space-time. The topology 3 depends very much on both fields, and there is only a little freedom left (which will become 
even clearer in $\S 5$; cf. Corollary 5.10). World lines - defined by 3 -continuity instead of $\mathfrak{I}$-continuity - are in fact physically realistic world lines, namely the orbits of charged test particles of positive rest mass under the action of the gravitational and the electro-magnetic field with a finite number of collisions with possible exchanges of mass and charge. There is an order preserving homeomorphism $h$ of $\left[\mathbf{0 , 1 ]}\right.$ such that $f^{*}=f \circ h$ is differentiable almost everywhere. We would like to point out that there are many unphysical "world lines" if one defines them by $\mathfrak{I}$-continuity.

(C) There are many more Zeeman topologies besides the Zeeman topologies (B) defined by special systems $\Sigma$, which are interesting for general relativity:

$\Sigma=$ all time like continuous curves [suggested by Hawking, oral communication] or

$\Sigma=$ all $d$-times differentiable (time like) curves.

These topologies will be considered in a future paper. ${ }^{4}$

\section{§ 4. Further Properties of the Zeeman Topologies on Space-Times}

In the following we restrict ourselves to Zeeman topologies $3=3\left(\sum_{\boldsymbol{q}}^{\boldsymbol{m}}, \mathfrak{I}\right)$ on space-times $(\boldsymbol{M}, \mathfrak{I})$ of general relativity as defined by (B) $(\S 3)$ or explicitly:

A subset $\boldsymbol{Y}$ of $\boldsymbol{M}$ is open with respect to $3\left(\Sigma_{\boldsymbol{q}}^{\boldsymbol{m}}, \mathfrak{I}\right)$ if and only if $\boldsymbol{Y} \cap \boldsymbol{U}$ is open in $\left(\boldsymbol{U}, \boldsymbol{I}_{\boldsymbol{U}}\right)$ for the following subsets $\boldsymbol{U}$ of $\boldsymbol{M}$ :

(I) $\boldsymbol{U}$ is an arbitrary closed space like hypersurface ${ }^{5}$ contained in a simple region of $\boldsymbol{M}$.

(II) $\boldsymbol{U}$ is the world line of an arbitrary charged test particle $\not h$ (with $m_{\hbar} \in \boldsymbol{m}$, $\left.q_{\mu} \in \boldsymbol{q}\right)$ freely falling in the gravitational and the electro-magnetic field within a simple region of $\boldsymbol{M}$. The spectra $\boldsymbol{m}$ and $\boldsymbol{q}$ are finite or $\boldsymbol{q}=\{0\}$.

If $q=\{0\}$, condition (II) is equivalent to

(II $) \boldsymbol{U}$ is an arbitrary time like geodesic in a simple region of $\boldsymbol{M}$. If we replace (I) by the more symmetric form,

(I*) $\boldsymbol{U}$ is an arbitrary space like geodesic in a simple region of $\boldsymbol{M}$. The topology $3^{*}$ defined by $\left(\mathbf{I}^{*}\right)$ and $\left(\mathbf{I I}_{0}\right)$ is strictly finer than $3_{0}=3\left(\Sigma_{0}, \mathfrak{I}\right)$ defined by $(\mathbf{I})$ and $\left(\boldsymbol{I I}_{0}\right)$, as can be seen from Minkowski space $\boldsymbol{M}$ : Let $\boldsymbol{H}$ be a 2-dimensional space like plane in $\boldsymbol{M}$, and consider the subset $\mathscr{S}=\left\{P_{n}=\left(\frac{1}{n}, \sin \left(\frac{1}{n}\right)\right) ; n \in \mathbb{N}\right\}$ of $\boldsymbol{H}$ with respect to a cartesian coordinate system. Then $\boldsymbol{Y}=\boldsymbol{M} \backslash \mathscr{S}$ is $3^{*}$-open, since any straight line of Minkowski space contains at most two points of $\mathscr{S}$. But $\boldsymbol{Y}$ is not open with respect to $\mathcal{Z}_{0}$, since $(0,0) \in \boldsymbol{H}$ is clearly an accumulation point of $\mathscr{S}$ with respect to $3_{0}$.

Lemma 3.2 gives the possibility of constructing $3\left(\sum_{\boldsymbol{q}}^{\boldsymbol{m}}, \mathfrak{I}\right)$-neighbourhoods which are not $\mathfrak{I}$-open. Since we will need two further types of (similar) neighbourhoods we proceed as follows:

If $\boldsymbol{U}$ is a simple neighbourhood of $P$, we denote by $\mathfrak{I}_{\boldsymbol{U}}(P)=\mathfrak{I}_{\boldsymbol{U}}^{+}(P) \cup \mathfrak{I}_{\boldsymbol{U}}^{-}(P)$ the (I-open) causal domain of $P$ within $\boldsymbol{U}$. Then let be [cf. § 2]

(D) $\boldsymbol{U}^{*}(P)=\boldsymbol{U} \cap\left(\mathfrak{I}_{\boldsymbol{U}}(P) \cup \mathfrak{\Xi}_{\boldsymbol{U}}(P)\right)=\left(\boldsymbol{U} \backslash \mathfrak{C}_{\boldsymbol{U}}(P)\right) \cup\{P\}$.

$4 \quad$ Cf. footnote ${ }^{2}$ (p. 292).

5 The embedding should at least be a $\mathscr{C}^{1}$-map. 
The sets $\mathfrak{\Xi}_{\boldsymbol{U}}(P)$ and $\boldsymbol{U}^{*}(P)$ are neither open nor closed in $(\boldsymbol{M}, \mathfrak{I})$, since $P \in \mathfrak{\Xi}_{\boldsymbol{U}}(P), P \in \boldsymbol{U}^{*}(P) . \mathfrak{E}_{\boldsymbol{U}}(P)=\mathfrak{\Xi}_{\boldsymbol{U}}(P) \backslash\{P\}$ is, however, I-closed.

Lemma 4.1. The set $\boldsymbol{U}^{*}(P)$ defined by (D) is a $3\left(\sum_{\boldsymbol{q}}^{\boldsymbol{m}}, \mathfrak{I}\right)$-neighbourhood of $P$.

[This shows again that $3\left(\sum_{\boldsymbol{q}}^{\boldsymbol{m}}, \mathfrak{I}\right)$ is strictly finer than $\mathfrak{I}$.]

Proof. (I) If $\boldsymbol{W}$ is a space like closed hypersurface of $\boldsymbol{M}$ (contained in a simple region), then

(a) $\boldsymbol{W} \cap \boldsymbol{U}^{*}=\boldsymbol{W} \cap\left[\boldsymbol{U} \cap\left(\mathfrak{I}_{\boldsymbol{U}}(P) \cup \mathfrak{S}_{\boldsymbol{U}}(P)\right]=\left[\boldsymbol{W} \cap \boldsymbol{U} \cap \mathfrak{I}_{\boldsymbol{U}}(P)\right] \cup\left[\boldsymbol{W} \cap \boldsymbol{U} \cap \mathfrak{\Xi}_{\boldsymbol{U}}(P)\right]\right.$.

If $P \in \boldsymbol{W}$ then $\boldsymbol{W} \cap \boldsymbol{U} \cap \mathfrak{I}_{\boldsymbol{U}}(P)=\emptyset$ and $\boldsymbol{W} \cap \boldsymbol{U} \cap \mathfrak{\Xi}_{\boldsymbol{U}}(P)=\boldsymbol{W} \cap \boldsymbol{U}$, since $\boldsymbol{W}$ is space like. Therefore $\boldsymbol{W} \cap \boldsymbol{U}^{*}=\boldsymbol{W} \cap \boldsymbol{U}$ because of (a), and (I) is satisfied since $\boldsymbol{U} \in \mathfrak{T}$. If $P \notin \boldsymbol{W}$, then $\boldsymbol{W} \cap \boldsymbol{U} \cap \mathfrak{S}_{\boldsymbol{U}}(P)=\boldsymbol{W} \cap \boldsymbol{U} \cap \mathfrak{E}_{\boldsymbol{U}}(P)$ is $\boldsymbol{I}_{\boldsymbol{W}}$-open, and $\boldsymbol{W} \cap \boldsymbol{U} \cap \mathfrak{I}_{\boldsymbol{U}}(P)$ is $\mathfrak{I}_{\boldsymbol{W}}$-open since $\boldsymbol{U} \cap \mathfrak{I}_{\boldsymbol{U}}(P) \in \mathfrak{I}$. Therefore $\boldsymbol{W} \cap \boldsymbol{U}^{*}$ is $\mathfrak{I}_{\boldsymbol{W}}$-open because of (a), and (I) is satisfied.

(II) Let $\boldsymbol{G}$ be a compact time like line in a simple region defined by (II). Then

(b) $\boldsymbol{G} \cap \boldsymbol{U}^{*}=\left[\boldsymbol{G} \cap \boldsymbol{U} \cap \mathfrak{J}_{\boldsymbol{U}}(P)\right] \cup\left[\boldsymbol{G} \cap \boldsymbol{U} \cap \widetilde{\Xi}_{\boldsymbol{U}}(P)\right]$.

If $P \in \boldsymbol{G}$ then $\left[\boldsymbol{G} \cap \boldsymbol{U} \cap \mathfrak{S}_{\boldsymbol{U}}(P)\right]=P$ and $\left[\boldsymbol{G} \cap \boldsymbol{U} \cap \mathfrak{I}_{\boldsymbol{U}}(P)\right]=\boldsymbol{G} \cap \boldsymbol{U} \backslash\{P\}$. Therefore $\boldsymbol{G} \cap \boldsymbol{U}^{*}=\boldsymbol{G} \cap \boldsymbol{U}$ by (b) satisfies (II). If $P \notin \boldsymbol{G}$, then $\boldsymbol{G} \cap \boldsymbol{U} \cap \mathfrak{I}_{\boldsymbol{U}}(P)$ and $\boldsymbol{G} \cap \boldsymbol{U} \cap \boldsymbol{G}_{\boldsymbol{U}}(P)=$ $\boldsymbol{G} \cap \boldsymbol{U} \cap \mathfrak{E}_{\boldsymbol{U}}(P)$ are $\mathfrak{I}_{\boldsymbol{G}}$-open. Therefore (II) is satisfied by (b).

Corollary 4.2. The topology induced by $3\left(\sum_{\boldsymbol{q}}^{\boldsymbol{m}}, \mathfrak{I}\right)$ on a light cone is discrete.

Proof. Let be $P \in \boldsymbol{L}$, and $\boldsymbol{L}$ is a light cone. Then $\boldsymbol{U}^{*}(P)$ defined by (D) is a $3\left(\sum_{\boldsymbol{q}}^{\boldsymbol{m}}, \mathfrak{I}\right)$-neighbourhood of $P$ and we have [by (D)] $\boldsymbol{U}^{*}(P) \cap \boldsymbol{L}=P$. Therefore $P$ is open in $\boldsymbol{L}$ with the topology induced by 3 and $\boldsymbol{L}$ is discrete.

Lemma 4.3. Let $\mathscr{S}=\left\{P_{n} ; n \in \mathbb{N}\right\}$ be a sequence of points of a simple neighbourhood $\boldsymbol{U}=\boldsymbol{U}(P)$ of $\boldsymbol{M}$ with the following properties:

(a) $P \notin \mathscr{S}$, but $P_{n} \longrightarrow P$.

(b) $\overline{P P}_{n}$ is a space like geodesic for all $P_{n} \in \mathscr{S}$.

(c) We have $|\boldsymbol{W} \cap \mathscr{S}|<\infty$ for all closed space like hypersurfaces $\boldsymbol{W}$ in $\boldsymbol{U}$.

Then $\boldsymbol{U}^{\prime}=\boldsymbol{U}^{\prime}(P)=\boldsymbol{U} \backslash \mathscr{S}$ is a $3\left(\sum_{\boldsymbol{q}}^{\boldsymbol{m}}\right.$, $\left.\mathfrak{I}\right)$-neighbourhood of $P$.

Proof. Let $\boldsymbol{G}$ be a (w.l.g.) compact world line as defined by (II), and assume $|\boldsymbol{G} \cap \mathscr{S}|=\infty$. Then $P \in \boldsymbol{G}$ by (a). Therefore $P \ll_{\boldsymbol{U}} P_{n}$ or $P_{n} \ll_{\boldsymbol{U}} P$ for all (infinitely many) $P_{n} \in \boldsymbol{G} \cap \mathscr{S}$, since $\boldsymbol{G}$ is time like, which contradicts (b). Therefore $|\mathscr{S} \cap \boldsymbol{G}|<\infty$, and Lemma 4.3 follows from Lemma 3.1.

Examples of sets $\mathscr{S}$ satisfying (a)-(c) of Lemma 4.3: Take an arbitrary $\mathscr{C}^{1}$ curve in $\boldsymbol{U}$ which is light like at $P$ and space like everywhere else. Take $\mathscr{S}$ to be a countable point set along this curve approaching $P$ with respect to $\mathfrak{I}$.

\section{§ 5. The Group of All Homeomorphisms of a Space-Time}

Since we are interested in symmetries of the space-time in this section, we will restrict ourselves to the Zeeman topology $3_{0}$ defined by (I) and $\left(\mathbf{I I}_{0}\right)$ of $\S 4$. A map $f$ of the topological space $\boldsymbol{A}$ into the topological space $\boldsymbol{B}$ is an embedding of $\boldsymbol{A}$ onto $f \boldsymbol{A}$ if $f$ is a homeomorphism of $\boldsymbol{A}$ onto $f \boldsymbol{A}$ with respect to the topology induced by $\boldsymbol{A}$ on $f \boldsymbol{A}$. 
Lemma 5.1. Let $f$ be an embedding of the Riemannian manifold $\boldsymbol{R}$ of dimension $\geqq 2$ into the space-time $\left(\boldsymbol{M}, 3_{0}\right)$ with respect to the Zeeman topology $3_{0}$. If $Q \in \boldsymbol{R}$ and $P=f(Q)$, there are a neighbourhood $\boldsymbol{U}$ of $Q$ and a simple neighbourhood $\boldsymbol{V}$ of $P$ such that $f \boldsymbol{U} \subseteq \Xi_{V}(P)$.

Remark. If $\boldsymbol{R}$ is a 3-dimensional ball with its Euclidean topology and $\boldsymbol{M}=$ Minkowski space, Lemma 5.1 strengthens a result of Zeeman [38, p. 166, Lemma 3]. Lemma 5.1 is still valid if we replace $3_{0}$ by the topologies $3\left(\sum_{\boldsymbol{q}}^{\boldsymbol{m}}, \mathfrak{I}\right)$ defined in (B) $\S 3$.

Proof. The map $f$ is $\mathfrak{I}$-continuous, since it is $3_{0}$-continuous and $\mathfrak{I} \leqq 3_{0}$. There is a simple neighbourhood $\boldsymbol{V}$ of $P$ (cf. $\S 2$ ), and we can find a neighbourhood $\boldsymbol{W}$ of $Q$ such that $f \boldsymbol{W} \subseteq \boldsymbol{V}$. We choose a set $\mathfrak{U}=\left\{\boldsymbol{U}_{n} ; n \in \mathbb{N}\right\}$ of neighbourhoods $\boldsymbol{U}_{n}$ of $Q$ such that $\boldsymbol{U}_{n+1} \subset \boldsymbol{U}_{n} \subset \boldsymbol{W}$ for all $n \in \mathbb{N}$, and $\bigcap\left\{\boldsymbol{U}_{n} ; n \in \mathbb{N}\right\}=Q$. We will now show

(a) There is an $\boldsymbol{U}_{n} \in \mathfrak{U}$ such that $f \boldsymbol{U}_{n} \subseteq \Xi_{V}(P) \cup \mathfrak{C}_{V}(P)$, and assume (a) to be false. Then we can construct a sequence $\mathscr{S}$ of points $P_{i}$ with the following properties:

(b) $P \neq P_{i} \in f \boldsymbol{U}_{i}$ for all $i \in \mathbb{N}$.

(c) $\overline{P P}_{i}$ is time like for all $i \in \mathbb{N}$.

(d) If $\overline{P P}_{i} \subseteq \overline{P P}_{k}$ for some $i, k \in \mathbb{N}$ then $i=k$.

We assume $P_{i}$ to be constructed for all $i<n$. Since (a) is assumed to be false, there is a point $P^{\prime} \in f \boldsymbol{U}_{n}$ such that $P^{\prime} \notin \mathfrak{G}_{\boldsymbol{V}}(P) \cup \mathfrak{C}_{\boldsymbol{V}}(P)$. The set $\boldsymbol{V}$ is simple and $\overline{P P^{\prime}}$ must be time like, i.e. $P^{\prime} \in \mathfrak{I}_{\boldsymbol{V}}(P) \cap f \boldsymbol{U}_{n}$. Therefore the set $\mathfrak{I}_{\boldsymbol{V}}(P) \cap f \boldsymbol{U}_{n}$ is not empty and open in $\left(f \boldsymbol{R},\left(3_{0}\right)_{f \boldsymbol{R}}\right)$. The map $f$ is $3_{f \boldsymbol{R}}$-continuous, hence there is an open subset $\boldsymbol{G}$ of $\boldsymbol{U}_{n}$ such that $P^{\prime} \in f \boldsymbol{G} \cong \mathfrak{I}_{\boldsymbol{V}}(P) \cap f \boldsymbol{U}_{n}$. The set $\boldsymbol{R}=\bigcup\left\{\overline{P P}_{i} ; i \in \mathbb{N}, i<n\right\}$ consists of finitely many 1 -dimensional submanifolds of $\boldsymbol{V}$. Since $f$ is an embedding of the at least 2-dimensional manifold $\boldsymbol{G}(\subseteq \boldsymbol{R})$, the set $f \boldsymbol{G}$ cannot be contained in $\mathfrak{R}$. Therefore there is a point $P_{n} \in f G$ such that $P_{n} \notin \bigcup \mathfrak{K}$ and $(\mathrm{d})$ is satisfied for all $i \leqq n$. Since $P_{n} \in f \boldsymbol{G} \subseteq \mathfrak{I}_{\boldsymbol{V}}(P) \cap f \boldsymbol{U}_{n}$, conditions (b) and (c) hold. Therefore $\mathscr{S}$ exists. The set $\boldsymbol{U}^{*}=\boldsymbol{V} \backslash \mathscr{S}$ is now a $\boldsymbol{3}_{0}$-neighbourhood of $P$ by Lemma 3.2. Therefore

(e) $P_{n} \underset{30}{\longrightarrow} P$ (with respect to the Zeeman topology $3_{0}$ ).

From (b) we obtain $f^{-1}\left(P_{i}\right) \in \boldsymbol{U}_{i}$ for all $i \in \mathbb{N}$, and have $\bigcap_{n \in \mathbb{N}} \boldsymbol{U}_{n}=Q$ by choice of $\mathfrak{U}$. Therefore we have $f^{-1}\left(P_{i}\right) \rightarrow Q$ with respect to the manifold topology on $\boldsymbol{R}$. Since $f$ is $3_{0}$-continuous by assumption, we get $P_{i} \underset{30}{\longrightarrow} f(Q)=P$ which contradicts (e). Therefore (a) is shown.

With the same notation we will show next

(f) There is an $\boldsymbol{U}_{n} \in \mathfrak{U}$ such that $f \boldsymbol{U}_{n} \subseteq \Xi_{\boldsymbol{V}}(P)$, and again we assume (f) to be false. Because of (a) we may assume w.l.g. that $f \boldsymbol{U}_{n} \subseteq \mathfrak{S}_{V}(P) \cup \mathfrak{C}_{V}(P)$. Therefore we get $f \boldsymbol{U}_{n} \cap \mathfrak{C}_{V}(P) \neq P$ for all $n \in \mathbb{N}$. In particular there is a sequence $\mathscr{S}$ of points $P \neq P_{n} \in f U_{n} \cap \mathfrak{C}_{V}(P)$. Then $U^{*}(P)-$ defined by (D) $\S 4-$ is a $3_{0}$-neighbourhood of $P$ by Lemma 4.1 which contains none of the points $P_{n} \in \mathscr{S}$, since $P \neq P_{n} \in \mathfrak{C}_{V}(P)$. Therefore we get again (e). Since $f^{-1}\left(P_{n}\right) \in \boldsymbol{U}_{n}, \bigcap_{n \in \mathbb{N}}^{\prime} \boldsymbol{U}_{n}=Q$ and $f$ is $3_{0}$-continuous, we derive $P_{n} \longrightarrow \mathrm{3o} P$ with respect to $3_{0}$, which contradicts (e). Therefore (f) and Lemma 5.1 are proved.

In the following we denote by $\boldsymbol{M}^{\prime}=\left(\boldsymbol{M}^{\prime}, \mathfrak{I}^{\prime}\right)$ a second space-time with its Zeeman topology $\mathbf{3}_{0}^{\prime}$ defined by $(\mathbf{I})$ and $\left(\mathbf{I I}_{0}\right)$ of $\S 4$. There are two types of homeomorphisms namely from $\left(\boldsymbol{M}, \mathbf{Z}_{0}\right)$ onto $\left(\boldsymbol{M}^{\prime}, \mathbf{3}_{0}^{\prime}\right)$ and from $(\boldsymbol{M}, \mathfrak{I})$ onto $\left(\boldsymbol{M}^{\prime}, \mathfrak{I}^{\prime}\right)$ which we will call $\mathbf{3}$-homeomorphism and $\mathfrak{I}$-homeomorphism respectively. 
Lemma 5.2. Let $h$ be a 3-homeomorphism from $\boldsymbol{M}$ onto $\boldsymbol{M}^{\prime}$. If $\boldsymbol{G}$ is a time like geodesic of $\boldsymbol{M}$ and $P \in \boldsymbol{G}$, there is a simple neighbourhood $\boldsymbol{V}$ of $P$ and a point $Q \in \mathfrak{I}_{\boldsymbol{V}}^{+}(P) \cap \boldsymbol{G}$ such that $h(\overline{P Q})$ is part of a time like geodesic of $\boldsymbol{M}^{\prime}$..

Proof. Let $i$ be the identical map from $\left(\boldsymbol{M}^{\prime}, \mathbf{Z}_{0}^{\prime}\right)$ onto $\left(\boldsymbol{M}^{\prime}, \mathfrak{I}^{\prime}\right)$. Then $i$ is continuous, but $i^{-1}$ is certainly not. Therefore $h^{*}=i \circ h$ is a continuous map from $\left(\boldsymbol{M}, \mathbf{3}_{0}\right)$ onto $\left(\boldsymbol{M}^{\prime}, \mathfrak{I}^{\prime}\right)$. Since $\left(\mathbf{3}_{0}\right)_{\boldsymbol{G}}=\mathfrak{I}_{\boldsymbol{G}}$ for time like geodesics $\boldsymbol{G}$ in simple neighbourhoods, there are (cf. $\S 2$ ) a simple neighbourhood $\boldsymbol{V}^{\prime}$ of $h(P) \in \boldsymbol{M}^{\prime}$ and a simple neighbourhood $\boldsymbol{V}$ of $P \in \boldsymbol{M}$ such that $h(\boldsymbol{V} \cap \boldsymbol{G}) \subseteq \boldsymbol{V}^{\prime}$. We assume

(a) There is a point $Q \in \mathfrak{J}_{V}^{+}(P) \cap \boldsymbol{G}$ such that $h(\overline{P Q})$ is a part of a geodesic of $\boldsymbol{M}^{\prime}$. To be false and construct a sequence $\mathscr{S}$ of points $P_{n} \in \mathfrak{I}_{V}^{+}(P) \cap G$ with the following properties:

(b) $h\left(P_{n}\right) \in V^{\prime}$ (which is simple).

(c) If $\overline{h(P) h\left(P_{n}\right)} \subseteq \overline{h(P) h\left(P_{m}\right)}$ for some $n, m \in \mathbb{N}$, then $m=n$.

(d) $P_{n} \underset{30}{\longrightarrow} P$ with respect to $3_{0}$.

We choose a set $\mathfrak{U}=\left\{\boldsymbol{U}_{n} ; n \in \mathbb{N}\right\}$ of $\mathfrak{I}$-neighbourhoods of $P$ such that $\boldsymbol{U}_{n+1} \subset \boldsymbol{U}_{n} \subset \boldsymbol{V}$ for all $n \in \mathbb{N}$ and $\bigcap_{n \in \mathbb{N}} \boldsymbol{U}_{n}=P$. We assume $P_{1}, \ldots, P_{n}$ to be constructed such that $P_{i} \in \boldsymbol{U}_{i}$. If there is no such $P_{n+1} \in \boldsymbol{U}_{n+1}$, this would mean $h\left(\overline{P P}_{n} \cap \boldsymbol{U}_{n+1}\right)$ is part of one of the geodesics through $h(P)$ and $h\left(P_{i}\right)$ for $i<n$. But this contradicts our assumption that (a) is false. Therefore $\mathscr{S}$ exists and satisfies (b), (c) and (d) since $P_{i} \in \boldsymbol{U}_{i}$ and $\bigcap_{n \in \mathbb{N}} \boldsymbol{U}_{n}=P$. We can choose an infinite subset of $\mathscr{S}$ so that we may assume w.l.g. all geodesics $\overline{h(P) h\left(P_{n}\right)}$ for $P_{n} \in \mathscr{S}$ are light like or time like or space like. If $\mathscr{S}^{\prime}=\left\{h\left(P_{n}\right) ; n \in \mathbb{N}\right\}$, then $\boldsymbol{U}^{*}=\boldsymbol{U}^{*}(h(P))$ - defined by (D), $\S 4$, if $\boldsymbol{U}=\boldsymbol{V}^{\prime}-$ is a $3_{0}^{\prime}$-neighbourhood of $h(P)$ with $\boldsymbol{U}^{*} \cap \mathscr{S}^{\prime}=\emptyset$ in the first case by Lemma 4.1. The set $\boldsymbol{U}^{*}=\boldsymbol{V}^{\prime} \backslash \mathscr{S}^{\prime}$ is a $3_{0}^{\prime}$-neighbourhood of $h(P)$ with $\mathscr{S}^{\prime} \cap \boldsymbol{U}^{*}=\emptyset$ in the second case by Lemma 3.2. Therefore $h\left(P_{n}\right)+_{30} h(P)$ for light like and time like geodesics, which contradicts (d) since $h$ is an 3-homeomorphism. Therefore we can assume

(e) All geodesics $\overline{h(P) h\left(P_{n}\right)}$ are space like for $P_{n} \in \mathscr{S}$. Next we want to show

(f) We have $\left|\mathscr{S}^{\prime} \cap \boldsymbol{W}\right|<\infty$ for all closed space like hypersurfaces $\boldsymbol{W}$ contained in simple regions of $\boldsymbol{M}^{\prime}$.

We assume $\left|\mathscr{S}^{\prime} \cap \boldsymbol{W}\right|=\infty$ for such a space like hypersurface $\boldsymbol{W}$. Then $h(P) \in \boldsymbol{W}$, since $\boldsymbol{W}$ is closed and we have

(g) $\left|\boldsymbol{U} \cap \mathscr{S}^{\prime}\right|=\infty$ for all neighbourhoods $\boldsymbol{U}$ of $h(P)$ in $\boldsymbol{W}=\left(\boldsymbol{W}, \mathfrak{I}_{\boldsymbol{W}}\right)$.

By Lemma 5.1 there is a neighbourhood $\boldsymbol{U}^{\prime}$ of $h(P)$ in $\boldsymbol{W}=\left(\boldsymbol{W}, \mathfrak{I}_{\boldsymbol{W}}\right)$ such that $h^{-1}\left(\boldsymbol{U}^{\prime}\right) \subseteq \Xi_{\boldsymbol{V}}(P)$. Since $\boldsymbol{G}$ is time like and $P \notin \mathscr{S} \subset \boldsymbol{G}$, it follows that $P=h^{-1}\left(\boldsymbol{U}^{\prime}\right) \cap \boldsymbol{G}$ and $\emptyset=h^{-1}\left(\boldsymbol{U}^{\prime}\right) \cap \mathscr{S}$. Therefore we get $\emptyset=\boldsymbol{U}^{\prime} \cap \mathscr{S}^{\prime}$, which contradicts (g), and (f) is shown.

Hence $V^{\prime} \backslash \mathscr{S}^{\prime}$ is a $3_{0}^{\prime}$-neighbourhood of $h(P)$ as follows by Lemma 4.3 together with (b), (d), (e), and (f). Therefore $h\left(P_{n}\right)+\underset{30}{+} h(P)$ also in this final case, which contradicts (d), hence (a) is shown. The set $h(\overline{P Q})$ cannot be contained in a light like geodesic, since its induced topology $3_{h(\overline{P Q})}$ would be discrete by Corollary 4.2 , but $(\overline{P Q}, 3 \overline{P Q})$ is connected, which is impossible for homeomorphisms $h$. We assume $h(\overline{P Q})$ to be space like. The space like geodesic $h(\overline{P Q})$ is contained in a closed space like hypersurface $\boldsymbol{W}$ which is contained in a simple region of $\boldsymbol{M}^{\prime}$. It follows that

(h) $|\boldsymbol{U} \cap h(\overline{P Q})|=\infty$ for all neighbourhoods $\boldsymbol{U}$ of $h(P)$ in $\boldsymbol{W}$.

By Lemma 5.1 there is a neighbourhood $\boldsymbol{U}^{\prime}$ of $h(P)$ in $\boldsymbol{W}$ such that $h^{-1}\left(\boldsymbol{U}^{\prime}\right) \subseteq \Xi_{\boldsymbol{V}}(P)$. Since $\overline{P Q}$ is time like, we get $P \in h^{-1}\left(\boldsymbol{U}^{\prime}\right) \cap \overline{P Q} \subseteq \Im_{\boldsymbol{V}}(P) \cap P Q=P$, 
i.e. $P=h^{-1}\left(\boldsymbol{U}^{\prime}\right) \cap \overline{P Q}$ or $h(P)=\boldsymbol{U}^{\prime} \cap h(\overline{P Q})$, which contradicts (h). Therefore $h(\overline{P Q})$ is time like, and Lemma 5.2 is proved.

Corollary 5.3. Let $h$ be a 3-homeomorphism from $\boldsymbol{M}$ onto $\boldsymbol{M}^{\prime}$. If $\boldsymbol{G}$ is a time like geodesic of $\boldsymbol{M}$ and $\boldsymbol{P} \in \boldsymbol{G}$, there is a point $P \neq Q \in \boldsymbol{G}$ such that $\overline{P Q}$ and $\overline{h(P) h(Q)}$ are contained in simple regions and we have $h(\overline{P Q})=\overline{h(P) h(Q)}$ a time like geodesic of $\boldsymbol{M}^{\prime}$.

Let $f$ be a map from the space-time $\boldsymbol{M}$ into the space-time $\boldsymbol{M}$. If $\boldsymbol{V}$ is contained in a simple region $\boldsymbol{U}$ of $\boldsymbol{M}$ and $f \boldsymbol{V}$ is contained in a simple region $\boldsymbol{U}^{\prime}$ of $\boldsymbol{M}^{\prime}$, then $f$ will be called orthochronal on $\boldsymbol{V}$ if $f(X) \ll_{\boldsymbol{U}^{\prime}} f(Y)$ for all $X, Y \in V$ with $X \ll_{\boldsymbol{U}} Y$, and anti-orthochronal on $\boldsymbol{V}$ if $f(Y) \ll_{\boldsymbol{U}^{\prime}} f(X)$ for all $X, Y \in \boldsymbol{V}$ with $X \ll_{\boldsymbol{U}} Y$. The 1-1-map $f$ of $\boldsymbol{M}$ onto $\boldsymbol{M}^{\prime}$ is then locally (anti-) orthochronal if $f$ and $f^{-1}$ are (anti-) orthochronal on any simple region of $\boldsymbol{M}$ or $\boldsymbol{M}^{\prime}$. The different $\ll$-related maps satisfy the following lemma, which will be applied to prove our main theorem:

Lemma 5.4. (Göbel $[13$, p. $5.6(\mathrm{~h})])$. A map is (locally) causal if and only if it is (locally) orthochronal or (locally) anti-orthochronal.

Lemma 5.5. Let $h$ be a 3-homeomorphism of the space-time $\boldsymbol{M}$ onto the spacetime $\boldsymbol{M}^{\prime}$. If $\boldsymbol{G}$ and $\boldsymbol{H}$ are two time like geodesics of $\boldsymbol{M}$ with a point $\boldsymbol{P}$ in common (which is not end point of $\boldsymbol{G}$ or $\boldsymbol{H}$ ), there are simple neighbourhoods $\boldsymbol{V}$ of $\boldsymbol{P}$ and $\boldsymbol{V}^{\prime}$ of $h(P)$ and points $Q \in \boldsymbol{G} \cap \mathfrak{I}_{V}^{+}(P)$ and $R \in \boldsymbol{H} \cap \mathfrak{I}_{V}^{+}(P)$ [analogous $R \in \boldsymbol{H} \cap \mathfrak{I}_{V}^{-}(P)$ ] with the following properties:

(1) We have $h(\overline{P Q})=\overline{h(P) h(Q)} \subset \boldsymbol{V}^{\prime}$ and $\overline{h(P R)}=\overline{h(P) h(R)} \subset \boldsymbol{V}^{\prime}$.

(2) The map $h$ is either orthochronal or anti-orthochronal on $\overline{P Q} \cup \overline{P R}$.

Proof. Let $\boldsymbol{V}^{\prime}$ be an arbitrary simple neighbourhood of $h(P)$. Since $\mathfrak{I}_{\mathbf{G}}=\left(\mathbf{3}_{0}\right)_{\mathbf{G}}$ and $\mathfrak{T}_{\boldsymbol{H}}=\left(\mathbf{Z}_{0}\right)_{\boldsymbol{H}}$, and $h$ is a 3 -homeomorphism of $\boldsymbol{M}$, there is a simple neighbourhood $\boldsymbol{V}$ of $P$ such that

(a) $h(\boldsymbol{V} \cap(\boldsymbol{G} \cup \boldsymbol{H})) \subset \boldsymbol{V}^{\prime}$.

We choose now $Q \in \boldsymbol{G} \cap \mathfrak{I}_{\boldsymbol{V}}^{+}(P)$ and w.l.g. $R \in \boldsymbol{H} \cap \mathfrak{I}_{\boldsymbol{V}}^{+}(P)$ according to Corollary 5.3. Therefore (1) is satisfied if we apply (a). The topology $\left(3_{0}\right)_{\overline{P Q}}$ and $\left(3_{0}^{\prime}\right)_{\overline{h(P) h(Q)}}$ is the Euclidean topology, and therefore $h$ is an ordinary homeomorphism between intervals if restricted to $\overline{P Q}$. Therefore $h$ is orthochronal or anti-orthochronal on $\overline{P Q}$, and we assume w.l.g. that

(b) $h$ is orthochronal on $\overline{P Q}$,

and we also assume that

(A) $h$ is anti-orthochronal on $\overline{P R}$.

There is a space like hypersurface $\boldsymbol{W}$ in $\boldsymbol{V}^{\prime}$ which contains $h(P)$ but not on its boundary. By Lemma 5.1 there is a neighbourhood $\boldsymbol{U}$ of $h(P)$ in $\boldsymbol{W}$ such that

(c) $h^{-1}(\boldsymbol{U}) \cong \Xi_{\boldsymbol{V}}(P), \boldsymbol{U}$ is a neighbourhood of $h(P)$ in $\boldsymbol{W}$, if we take $h^{-1}$ restricted to $\boldsymbol{W}$ to be $f$. Since $h(P)$ is not at the boundary of $\boldsymbol{W}$, it is contained in a Euclidean " $\varepsilon$ "-neighbourhood $\boldsymbol{E}$ of $\boldsymbol{W}$. Because $\boldsymbol{E}$ is open in a space like hypersurface we can choose a 3-simplex $\Delta^{3}$ contained in $\boldsymbol{E}\left(\subseteq \boldsymbol{V}^{\prime}\right.$ which is simple) with barycentre $h(P)$. Since $h$ restricted to $\overline{P Q}$ is continuous in the ordinary (Euclidean) sense, we can choose a point $Q^{\prime} \in \overline{P Q}$ sufficiently close to $P$ such that the 4-simplex $\Delta^{4}$ generated by $\Delta^{3}$ and $h\left(Q^{\prime}\right)$ has faces, which are contained in space like hypersurfaces. Therefore the topology, induced by $3_{0}^{\prime}$ at its boundary $\partial \Delta^{4}$ coincides with the topology 
induced by $\mathfrak{I}^{\prime}$. Therefore $\left(\partial \Delta^{4},\left(3_{0}^{\prime}\right)_{\partial \Delta^{4}}\right)=\left(\partial \Delta^{4},\left(\mathfrak{I}^{\prime}\right)_{i \Delta^{4}}\right)$ is homeomorphic to a 3 -sphere and is compact. We now consider the map $f=i \circ h_{0}^{-1}$, where $h_{0}^{-1}$ is $h^{-1}$ restricted to $\partial \Delta^{4}$ and $i$ the identical map from $\left(\boldsymbol{M}, \mathbf{Z}_{0}\right)$ onto $(\boldsymbol{M}, \mathfrak{T})$. The map $f$ is a 1-1-map of a compact space into the Hausdorff space $(\boldsymbol{M}, \mathfrak{I})$ and is continuous, since $\mathfrak{I} \leqq \mathfrak{Z}_{0}$. Therefore $f$ is a $\mathfrak{I}$-homeomorphism; cf. Kelley [19, p. 141, Theorem 8]. Let $\mathfrak{B}$ be a $\mathfrak{I}$-neighbourhood basis of $P$. Since $f$ is an embedding, the set $f^{-1} \mathfrak{B}=\left\{f^{-1}(S) \cap \partial \Delta^{4}, S \in \mathfrak{B}\right\}$ is a neighbourhood basis of $h(P)$ in $\partial \Delta^{4}$. Therefore there is an $\boldsymbol{U}^{\prime} \in \boldsymbol{B}$ such that $f^{-1}\left(\boldsymbol{U}^{\prime}\right) \cap \partial \Delta^{4} \subseteq \boldsymbol{U}$. We apply $h^{-1}$ to this inequality and obtain $\boldsymbol{U}^{\prime} \cap h^{-1}\left(\partial \Delta^{4}\right) \subseteq h^{-1}(\boldsymbol{U})$, and with (c) we get $\boldsymbol{U}^{\prime} \cap h^{-1}\left(\partial \Delta^{4}\right) \cap \mathfrak{I}_{\boldsymbol{V}}(P) \subseteq h^{-1}(\boldsymbol{U}) \cap \mathfrak{I}_{\boldsymbol{V}}(P)=\emptyset$, i.e. $\boldsymbol{U}^{\prime} \cap h^{-1}\left(\partial \Delta^{4}\right) \cap \mathfrak{I}_{\boldsymbol{V}}(P)=\emptyset$. Therefore we have

(d) $\left(\boldsymbol{U}^{\prime} \cap \mathfrak{I}_{\boldsymbol{V}}^{+}(P)\right) \backslash h^{-1}\left(\partial \Delta^{4}\right)=\boldsymbol{U}^{\prime} \cap \mathfrak{I}_{\boldsymbol{V}}^{+}(P)$.

Let $\boldsymbol{C}$ be the set of all points $X \in \boldsymbol{V}^{\prime}$ which can be connected by a $3_{0}$-connected curve with $\left(\overline{h(P) h\left(Q^{\prime}\right)}\right)$ within $\boldsymbol{V}^{\prime} \backslash \partial \Delta^{4}$. Then $\boldsymbol{C}$ consists of the interior of $\Delta^{4}$, because each inner point of $\Delta^{4}$ can be connected by a piecewise geodesic line which is space or time like. But this curve is $3_{0}$-connected, since its $3_{0}$-induced topology coincides with the $\mathfrak{I}$-induced topology. From assumption (A) we get

(e) $(\overline{h(P) h(R)}) \cap C=\emptyset$.

Since $U^{\prime}$ is a $\mathfrak{I}$-neighbourhood of $P$ and $(\overline{P R}) \cup\left(\overline{P Q^{\prime}}\right) \subseteq \mathfrak{J}_{V}(P)$, there are points on $(\overline{P R})$ and $\left(\overline{P Q^{\prime}}\right)$ which can be connected by a (space like) curve lying entirely in $\boldsymbol{U}^{\prime} \cap \mathfrak{I}_{V}^{+}(P)$ and in a space like hypersurface. This curve is $3_{0}$-continuous, and

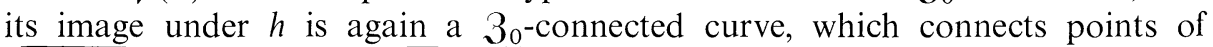
$(\overline{h(P) h(R}))$ with points of $\left(\overline{h(P) h\left(Q^{\prime}\right)}\right)$ and is in $V^{\prime} \backslash \partial \Delta^{4}$ because of $(\mathrm{d})$. Therefore

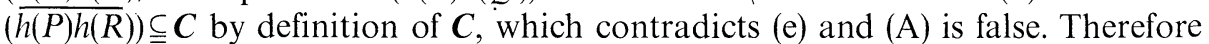
Lemma 5.5 is proved.

Corollary 5.6. Let $\boldsymbol{G}$ be a compact [part of $(a)]$ time like geodesic of a spacetime $\boldsymbol{M}$ and $h$ be a 3 -homeomorphism of $\boldsymbol{M}$ onto a space-time $\boldsymbol{M}^{\prime}$. Then $h(\boldsymbol{G})$ is time like and piecewise geodesic and $h$ is (locally) orthochronal or anti-orthochronal on $\boldsymbol{G}$.

Proof. If $P \in G$, there are a simple neighbourhood $\boldsymbol{V}$ of $P$ and points $Q \in \mathfrak{I}_{\boldsymbol{V}}^{+}(P) \cap \boldsymbol{G}$ (if $P$ is the future end point of $\boldsymbol{G}$ we put $Q=P$ ) and $R \in \mathfrak{I}_{\boldsymbol{V}}^{-}(P) \cap \boldsymbol{G}$ (if $P$ is the past end point of $G$ we put $R=P$ ) such that

$$
h(\overline{R Q})=h(\overline{R P} \cup \overline{P Q})=h(\overline{R P}) \cup h(\overline{P Q})=\overline{h(R) h(P)} \cup \overline{h(P) h(Q)}
$$

where $\overline{h(R) h(P)}, \overline{h(P) h(Q)}$ are time like geodesics according to Lemma 5.5 (1). Since $G$ is compact, it can be covered by finitely many such segments $\overline{R Q}$. Therefore $h(\boldsymbol{G})$ is a piecewise geodesic line and time like. If $\overline{R Q}$ is again such a segment of the finite covering, $h$ is (locally) orthochronal or anti-orthochronal on $\overline{R Q}$ by Lemma 5.5 (2). We assume w.l.g. $h$ to be orthochronal on $\overline{R Q}$. If we apply Lemma 5.5 (2) to all other connected segments of the finite covering, we derive that $h$ is (locally) orthochronal on $\boldsymbol{G}$.

Corollary 5.7. A 3-homeomorphism $h$ of a space-time $\boldsymbol{M}$ onto a space-time $\boldsymbol{M}^{\prime}$ has the following properties:

(1) $h$ is a causal map.

(2) $h$ is a I-homeomorphism.

(3) $h$ is locally causal. 
Proof. (1) It is sufficient to show

(a) We have $h(X) \ll h(Y)$ or $h(Y) \ll h(X)$ if $X \ll Y$ for $X, Y \in M$.

Since $X \ll Y$, there is a differentiable, time like, future directed curve connecting $X$ with $Y$. Since this curve is compact, there are points $P_{1}, \ldots, P_{n}$ (on this curve) in $M$ such that $\bar{P}_{i} P_{i+1}$ are future directed, time like geodesics in simple regions for $1 \leqq i<n$ and $P_{1}=X, P_{n}=Y$; cf. Penrose [25, p. 15, Proposition 2.23]. We denote this piecewise geodesic line by $\boldsymbol{G}$. Then $h\left(\bar{P}_{i} P_{i+1}\right)$ is time like and piecewise geodesic by Corollary 5.6. Therefore $h(\boldsymbol{G})$ is again piecewise geodesic and time like. By application of Corollary 5.6 and Lemma 5.5 follows that $h$ is orthochronal or anti-orthochronal on $\boldsymbol{G}$. A second application of Penrose [25, p. 15, Proposition 2.23] then shows that $h(X)$ can be connected with $h(Y)$ by a differentiable, time like either future or past directed curve. Hence $h(X) \ll h(Y)$ or $h(Y) \ll h(X)$.

The topology on $\boldsymbol{M}$ generated by the (I-open) subsets $\mathfrak{I}^{-}(Q) \cap \mathfrak{I}^{+}(P)$ for all $P, Q \in M$ is called Alexandrov topology. In general $\mathfrak{I}$ is finer than the Alexandrov topology as we know from "cyclinder worlds". A space-time $\boldsymbol{M}$ is then strongly causal (in the sense of Hawking [15]) if its Alexandrov topology coincides with its manifold topology $\mathfrak{I}$; more physical (equivalent) definitions of strongly causal may be found in Penrose [25, p. 34, Theorem 4.24].

(2) If $\boldsymbol{M}$ and $\boldsymbol{M}^{\prime}$ are strongly causal, property (2) of Corollary 5.7 follows from (1): Since $h$ is a causal map by (1), it maps Alexandrov-neighbourhoods onto Alexandrov-neighbourhoods as follows from Lemma 5.4 and the definition of Alexandrov topology. Since $\boldsymbol{M}$ and $\boldsymbol{M}^{\prime}$ are strongly causal $h$ maps $\mathfrak{I}$ onto $\mathfrak{I}^{\prime}$. Hence $h$ is a $\mathfrak{I}$-homeomorphism.

In general we use a method which has some similarity with the proof of Lemma 5.5. First we show that it is sufficient to prove

(b) If $P \in \boldsymbol{U} \in \mathfrak{T}$, there is a set $\boldsymbol{V} \subseteq \boldsymbol{U}$ such that $P \in \boldsymbol{V}$ and $h(\boldsymbol{V}) \in \mathfrak{I}^{\prime}$.

We denote $\boldsymbol{V}=\boldsymbol{V}(P)$. Then we have $\boldsymbol{U}=\bigcup_{\boldsymbol{P} \in \boldsymbol{U}} \boldsymbol{V}(P)$ and therefore $h(\boldsymbol{U})=$ $h\left(\bigcup_{P \in \boldsymbol{U}} \boldsymbol{V}(P)\right)=\bigcup_{P \in \boldsymbol{U}} h(\boldsymbol{V}(P)) \in \boldsymbol{I}^{\prime}$ by a property of topology and (b). We get $h(\mathfrak{I}) \subseteq \mathfrak{I}^{\prime}$. Because of the symmetry (for $h^{-1}$ ) we obtain $h^{-1}\left(\mathfrak{I}^{\prime}\right) \subseteq \mathfrak{I}$ and together $h(\mathfrak{I})=\mathfrak{I}^{\prime}$, which means $h$ is a $\mathfrak{I}$-homeomorphism.

Let be $P \in \boldsymbol{U} \in \mathfrak{T}$. Like in the proof of Lemma 5.5 we construct a 4-simplex $\Delta^{4} \subseteq \boldsymbol{U}$ such that its boundary $\boldsymbol{D}=\left(\boldsymbol{D},\left(3_{0}\right)_{\boldsymbol{D}}\right)$ is homeomorphic to a 3 -sphere $S^{3}$ with respect to the topology induced by $3_{0}$ and such that $P$ is an interior point $P \in \operatorname{int}(\boldsymbol{D})$. We consider the restriction $h^{*}$ of $h$ mapping $\left(\boldsymbol{D},\left(\boldsymbol{3}_{0}\right)_{\boldsymbol{D}}\right)$ into $\left(\boldsymbol{M}^{\prime}, \boldsymbol{I}^{\prime}\right)$. The map $h^{*}$ is a continuous $1-1$-map sending the compact space $\left(\boldsymbol{D},\left(\boldsymbol{3}_{0}\right)_{\boldsymbol{D}}\right) \simeq \mathrm{S}^{3}$ into the Hausdorff space $\left(\boldsymbol{M}^{\prime}, \mathfrak{I}^{\prime}\right)$. Therefore $h^{*}$ is an embedding by Kelley [19, p. 141, Theorem 8], i.e.

(c) $h(\boldsymbol{D})=\boldsymbol{D}^{\prime}$ is a 3 -sphere: $\left(\boldsymbol{D}^{\prime},\left(\mathfrak{I}^{\prime}\right)_{\boldsymbol{D}^{\prime}}\right) \simeq \mathrm{S}^{3}$.

We consider now the homeomorphism $h_{0}$ from $\left(\boldsymbol{M} \backslash \boldsymbol{D},\left(\boldsymbol{3}_{0}\right)_{\boldsymbol{M} \backslash \boldsymbol{D}}\right)$ onto $\left(\boldsymbol{M}^{\prime} \backslash \boldsymbol{D}^{\prime},\left(\mathbf{3}_{0}^{\prime}\right)_{\boldsymbol{M}^{\prime} \backslash \boldsymbol{D}^{\prime}}\right)$. It maps the $\mathbf{3}_{0}$-connected component $\boldsymbol{C}_{\mathbf{3}_{0}}(P)$ of $P$ onto the $3_{0}^{\prime}$-connected component $\boldsymbol{C}_{30^{\prime}}\left(P^{\prime}\right)$ of $P^{\prime}=h(P)$ :

(d) $h\left(\boldsymbol{C}_{30}(P)\right)=\boldsymbol{C}_{30}\left(P^{\prime}\right)$.

Any two inner points of $\boldsymbol{D}$ can be connected by a piecewise geodesic line of time like and space like geodesics, i.e. by a $3_{0}$-connected curve. Hence $\operatorname{int}(\boldsymbol{D})=$ $\boldsymbol{C}_{30}(P)$, and the same holds for $\boldsymbol{D}^{\prime}$ because of (c). If $P^{\prime}$ is an inner point of $\boldsymbol{D}^{\prime}$ we have $C_{30}\left(P^{\prime}\right)=\operatorname{int}\left(\boldsymbol{D}^{\prime}\right) \in \mathfrak{T}^{\prime}$ (the argument is similar if $P^{\prime}$ is an outer point of $\boldsymbol{D}^{\prime}$ ). 
Together with (d) we derive $\boldsymbol{U} \supseteqq \operatorname{int}(\boldsymbol{D})=\boldsymbol{C}_{30}(P) \ni P$ and $h\left(\boldsymbol{C}_{30}(P)\right)=\boldsymbol{C}_{30}\left(P^{\prime}\right)=$ $\operatorname{int}\left(\boldsymbol{D}^{\prime}\right) \in \mathfrak{I}^{\prime}$. We put $\boldsymbol{V}=\boldsymbol{C}_{3_{0}}(P)$, and (b) is proved.

(3) There are simple neighbourhoods $\boldsymbol{V}$ of $P$ and $\boldsymbol{V}^{\prime}$ of $h(P)$ such that $h(\boldsymbol{V}) \subseteq \boldsymbol{V}^{\prime}$, which follows from Corollary 5.7 (2). We consider the restricted homeomorphism between $\boldsymbol{V}$ and $h(\boldsymbol{V})$ and apply (1) to obtain (3).

Proposition 5.8. For a map $h$ of a space-time $\boldsymbol{M}$ onto a space-time $\boldsymbol{M}^{\prime}$ are equivalent:

(1) $h$ is a homothetic transformation.

(2) $\{$ (a) $h$ is a conformal transformation.

(b) h maps time like geodesics (as point sets) onto time like geodesics.

Proof. Let $h$ be a conformal map with conformal factor $\Omega=\Omega(X), X \in \boldsymbol{M}$; cf. $\S 2$. If $f: t \in[\mathbf{0}, \mathbf{1}] \rightarrow f(t) \in \boldsymbol{M}$ is a geodesic (with the differentiable not necessarily affine parameter $t$ ) with tangent vectors $K=\frac{\partial}{\partial t}$, then their coordinates $K^{a}$ satisfy the equation

(a) $K^{[b} \cdot \frac{\boldsymbol{D}}{\partial t} K^{a]}=0$

cf. Hawking and Ellis [16, p. 32, 33]. Let $f^{*}=h \circ f$ be the image curve of $f$ under the conformal transformation $h, \Omega^{*}$ its tangents along $f^{*}$ and $\boldsymbol{g}^{*}=\Omega^{2} \cdot \boldsymbol{g}$ the metric at the point $h(X)$ of $\boldsymbol{M}^{\prime}$ if $\boldsymbol{g}$ is the metric at $P \in \boldsymbol{M}$. According to Hawking and Ellis $\left[16\right.$, p. 63] the derivative $\frac{\boldsymbol{D}^{\prime}}{\partial t} \mathfrak{\Re}^{*}$ of $\mathfrak{\Omega}^{*}$ along $f^{*}$ can be expressed in terms of $\Omega$ :

$$
\frac{\boldsymbol{D}^{\prime}}{\partial t} K^{* a}=\frac{\boldsymbol{D}}{\partial t} K^{a}+2 \cdot\left(\nabla_{b} \log \Omega\right) \cdot K^{b} \cdot K^{a}-\left(K^{b} \cdot K^{c} \cdot g^{*}{ }_{b c}\right) \cdot g^{* a d} \nabla_{d} \log \Omega .
$$

Therefore we have

$$
K^{*[b} \cdot \frac{\boldsymbol{D}^{\prime}}{\partial t} K^{* a]}=K^{[b} \cdot \frac{\boldsymbol{D}}{\partial t} K^{a]}-\left(K^{b} \cdot K^{c} \cdot g_{b c}^{*}\right) \cdot K^{[b} \cdot g^{* a] d} \cdot \nabla_{d} \log \Omega .
$$

From (a) we obtain

(b) $\left\{\begin{array}{l}K^{*[b} \cdot \frac{\boldsymbol{D}^{\prime}}{\partial t} K^{* a]}=-\left(\Omega^{2} \cdot\|\mathfrak{R}\|\right)^{2} \cdot K^{[b} \cdot \nabla^{a]} \log \Omega \\ \text { if } f \text { is a geodesic where }\|\mathfrak{\Re}\|^{2}=K^{a} \cdot K^{b} \cdot g_{a b} .\end{array}\right.$

(1) $\rightarrow(2)$ : If $h$ is a homothetic transformation, this map is by definition a conformal transformation and in particular we have $\Omega=$ const. Therefore we get $\nabla_{b}(\log \Omega)=0$, and from (b) it follows that $K^{*[b} \cdot \frac{D^{\prime}}{\partial t} K^{* a]}=0$, if $f$ is a geodesic. Therefore $f^{*}$ is a geodesic, and (2)(a) and (2)(b) are derived from (1).

(2) $\rightarrow(1)$ : From assumption (2) and (b) follows that $\left(\Omega^{2} \cdot\|\mathfrak{R}\|\right)^{2} \cdot K^{[b} \cdot \nabla^{a]} \log \Omega=0$ for time like geodesics. Since $\|\boldsymbol{\Omega}\|>0$ and $\Omega \neq 0$ we obtain $K_{[b} \cdot \nabla_{a]} \log \Omega=0$. The left hand side of this equation represents a skew vector of a singular surface element; cf. Jordan, Ehlers and Kundt [18, p. 31]. Therefore the generating vectors are parallel, i.e. 
(c) $K_{a} \sim \nabla_{a} \log \Omega$.

The right hand side of (c) represents a fixed direction at a point. At the same time the left hand side of (c) can be chosen to be an arbitrary time like direction. Therefore $\nabla_{a} \log \Omega=0$ and $\log \Omega$ as well as $\Omega$ are constant along time like geodesic lines. A space-time $\boldsymbol{M}$ is connected (by definition) and therefore two arbitrary points of $\boldsymbol{M}$ can be connected by a piecewise geodesic which is time like. Therefore $\Omega$ is constant throughout the whole space-time $\boldsymbol{M}$, and $h$ is homothetic.

Main Theorem 5.9. For a map $h$ of a space-time $\boldsymbol{M}$ onto a space-time $\boldsymbol{M}^{\prime}$ are equivalent:

(1) $h$ is a homeomorphism with respect to the Zeeman topology $3_{0}$ [defined by (I) and ( $\left.\mathbf{I I}_{0}\right)$ in $\$ 47$.

(2) $h$ is a homothetic transformation.

Remark. This is the full solution to a question raised by Zeeman [38, p. 162, problem (1)].

Proof. (1) $\rightarrow$ (2). A 3 -homeomorphism $h$ of $\boldsymbol{M}$ onto $\boldsymbol{M}^{\prime}$ is a $\mathfrak{T}$-homeomorphism (with respect to the manifold topology $\mathfrak{T}$ ) as follows from Corollary 5.7 (2). Therefore for each point $P \in \boldsymbol{M}$ there is a simple neighbourhood $\boldsymbol{V}^{\prime}$ of $h(P) \in \boldsymbol{M}^{\prime}$ (cf. §2), and we can find a simple neighbourhood $\boldsymbol{V}$ of $P$ such that $h(\boldsymbol{V}) \leqq \boldsymbol{V}^{\prime}$. The restriction $h^{*}$ of $h$ to $\boldsymbol{V}$ is a 3 -homeomorphism of the space-time $\boldsymbol{V}$ onto the space-time $h(\boldsymbol{V})$ [which is a simple neighbourhood of $\boldsymbol{M}^{\prime}$ by Corollary 5.7 (2)]. The map $h^{*}$ is a causal map by Corollary 5.7 (1). Therefore $h^{*}$ is orthochronal or anti-orthochronal by Lemma 5.4. Since $\boldsymbol{V}$ and $h(\boldsymbol{V})$ are simple neighbourhoods of $\boldsymbol{M}$ and $\boldsymbol{M}^{\prime}$ respectively, the spaces $\boldsymbol{V}$ and $h(\boldsymbol{V})$ are strongly causal. Therefore $h^{*}$ is a diffeomorphism (degree of differentiability $=\min \left(d, d^{\prime}\right)-1 \geqq 2$; if $d$ and $d^{\prime}$ are the degrees of differentiability of $\boldsymbol{M}$ and $\boldsymbol{M}^{\prime}$ respectively) of $\boldsymbol{V}$ onto $h(\boldsymbol{V})$, which follows from Hawking [15, p. 116, Lemma 3] ${ }^{6}$ or Göbel [13, p. II.6.1, (a)]. Therefore $h^{*}$ is a conformal map of $\boldsymbol{V}$ onto $h(\boldsymbol{V})$ (cf. Göbel [13, p. II.6.9]), which follows by application of an argument in Hawking and Ellis [16, p. 61]. Using a covering of $\boldsymbol{M}$ by simple neighbourhoods of the type $\boldsymbol{V}$ we obtain that $h$ is a conformal map from $\boldsymbol{M}$ onto $\boldsymbol{M}^{\prime}$. Because of Corollary 5.6 and the differentiability of $h$ we derive that $h$ maps time like geodesics onto time like geodesics (there are no more edges!). The conformal map $h$ is now homothetic by Proposition 5.8. Therefore (2) is proved.

(2) $\rightarrow$ (1) follows by definition of $3_{0}$ and Proposition 5.8.

Discussion. In the main Theorem 5.9 we derive from the continuity of the (homeomorphism) $h$ with respect to the underlying Zeeman topology $\mathbf{3}_{0}$ its differentiability as well as its "linearity" since $h$ is [by (2)] an isometric map "up to scaling". This is a very unusual property of a topology, which we may put into a different and even more characteristic form:

Corollary 5.10. The space-times $\boldsymbol{M}$ and $\boldsymbol{M}^{\prime}$ are homeomorphic in its Zeeman topologies [defined by (I) and $\left(\mathbf{I I}_{0}\right)$ in $\S 4$ ] if and only if they are isometric (up to a constant positive factor).

Corollary 5.11. The group $\operatorname{Hom}\left(\boldsymbol{M}, \mathbf{3}_{0}\right)$ of all homeomorphisms with respect to the Zeeman topology coincides with the group $\operatorname{Hot}(\boldsymbol{M})$ of all homothetic transformations of the space-time $\boldsymbol{M}$ onto itself, i.e. $\operatorname{Hom}\left(\boldsymbol{M}, \mathbf{Z}_{0}\right)=\operatorname{Hot}(\boldsymbol{M})$.

Note Added in Proof. Lemma 3 will now be published in Hawking, King, McCarthy [42, Theorem 5]. 
Corollary 5.10 implies that Zeeman topology contains all informations about the metric; therefore the metric can be derived from this topology. Such a type of topology - or some of its properties - might be very useful for a reconstruction of general relativity in an axiomatic way. In particular this topology might be useful for approaches to this on a basis of projective geometry like in Ehlers and Schild [10] or Woodhouse [36]. In this sense it would be interesting to tackle the following problem: Consider a space-time $M$ with a metric $g$ and specify certain subsets of $\boldsymbol{M}$ to be world lines of free falling particles (= "time like geodesics"). Then introduce a topology $\mathbf{Z}^{\prime}$ on $\boldsymbol{M}$ which is Euclidean on the world lines of freely falling particles. Under what conditions can the metric be derived from $3^{\prime}$ (up to a constant factor)?

If $\boldsymbol{M}$ is the Minkowski space, Corollary 5.11 is the main result of Zeeman [38, p. 168, Theorem 3], since $\operatorname{Hot}(\boldsymbol{M})$ is the Weyl group of $M$ which is the semidirect product of dilatations and the Poincaré group $(=$ inhomogeneous Lorentz transformations) of Minkowski space. On the other hand there are space-times $\boldsymbol{M}$ (even vacuum solutions of Einstein's field equations; cf. Robinson and Robinson [28]) with a trivial conformal group. In particular we have $\operatorname{Hot}(\boldsymbol{M})=1$, i.e. the identity is the only homeomorphism of $\boldsymbol{M}$ onto itself with respect to the Zeeman topology. The freedom of choice of continuous maps is gone!

\section{§ 6. References}

1. Alexandrov, A. D.: A contribution to chronogeometry. Can. J. Math. 19, 1119-1128 (1967)

2. Alonso, J.L., Ynduráin, F. J.: On the continuity of causal automorphisms of space-time. Commun. math. Phys. 4, 349-351 (1967)

3. Barucchi, G.: The group of timelike permutations. Nuovo Cimento 55 A, 385-395 (1968)

4. Benz, W.: Zur Linearität relativistischer Transformationen. Jahresber. der DMV 70, 100-108 (1967)

5. Borchers, H.J., Hegerfeldt, G.C.: The structure of space-time transformations. Commun. math. Phys. 28, 259-266 (1972)

6. Brechner, B.: Topological groups which are not full homeomorphism groups. Duke Math. J. 39, 97-99 (1972)

7. Cel'nik, F.A.: Topological structure of space in relativistic mechanics. Dokl. Akad. Nauk SSSR 181 (1968); engl. transl.: Soviet Math. Dokl. 9, 1151-1152 (1968)

8. Domiaty, R.Z.: Verallgemeinerte metrische Räume. I. Der innere Abstand. Bericht 6 (1973) der Math-Stat. Sektion im Forschungszentrum Graz

9. Ehlers, J.: General relativity and kinetic theory. In: Sachs, R.K. (Ed.): Relatività generale a cosmologia. New York: Academic Press 1971

10. Ehlers, J., Schild, A.: Geometry in a manifold with projective structure. Commun. math. Phys. 32, $119-146(1973)$

11. Freudenthal, H.: Das Helmholtz-Liesche Raumproblem bei indefiniter Metrik. Math. Ann. 156, $263-312(1964)$

12. Gheorghe, C., Mihul, E.: Causal groups of space-time. Commun. math. Phys. 14, 165-170 (1969)

13. Göbel, R.: Die volle kausale Gruppe der Raum-Zeit, physikalischer Teil (II) der Habilitationsschrift, Würzburg 1973

14. Guts, A. K.: Mappings that preserve cones in Lobachevskii space. Matem. Zametki 13, 687-694 (1973) (russ.); engl. transl.: Consultants Bureau, 411-415 (1973); Plenum Publ. Co. New York, and Sov. Math. Doklady 15, 416-419 (1974)

15. Hawking, S. W.: Singularities and the geometry of space time. Adams Prize Essay, Cambridge 1966

16. Hawking, S.W., Ellis, G.F.R.: The large scale structure of space-time. Cambridge: University Press 1973

17. Hegerfeldt, G.C.: The Lorentz transformations: Derivation of linearity and scale factor. Nuovo Cimento 10 A, 257-267 (1972) 
18. Jordan, P., Ehlers, J., Kundt, W.: Strenge Lösungen der Feldgleichungen der allgemeinen Relativitätstheorie. Akad. der Wiss. und Literatur. Mainz 1960

19. Kelley, J. L.: General topology. Princeton N.J.: Van Nostrand 1955

20. Kobayashi, S., Nomizu, K.: Foundations of differential geometry. New York: Interscience Publ. 1963

21. Loewner, C.: On semigroups in analysis and geometry. Bull. AMS 70, 1-15 (1964)

22. Michél, L.: Relation entre symétries internes et invariance relativiste. In: Lurcat, F. (Ed.): Applications of mathematics to problems in theoretical physics, pp. 409-459. Gordon and Breach 1967

23. Nanda, S.: Topology for Minkowski space. J. Math. Phys. 12, 394-401 (1971)

24. Nevanlinna, R.: Raum, Zeit und Relativität. Stuttgart, Basel: Birkhäuser Verlag 1964

25. Penrose, R.: Techniques of differential topology in relativity. Philadelphia, Penn.: SIAM 1972

26. Rätz, J.: On isometries of generalized inner product spaces. SIAM J. Appl. Math. 18, 6—9 (1970)

27. Rothaus, O.S.: Order isomorphisms of cones. Proc. AMS 17, 1284-1288 (1966)

28. Robinson, I., Robinson, J.: Vacuum metrics without symmetry (unpublished) (1969)

29. Süßmann, G.: Begründung der Lorentz-Gruppe allein mit der Symmetrie und Relativitätsannahmen. Z. Naturforsch. 24a, 495-498 (1969)

30. Teppati, G.: An algebraic analogue of Zeeman's theorem. Nuovo Cimento 54, 800 - 804 (1968)

31. Vilms, J.: Totally geodesic maps. J. Diff. Geom. 4, 73-79 (1970)

32. Vock, V.: Theorie von Raum, Zeit und Gravitation. Berlin: Akademie Verlag 1960

33. Vogt, A.: Maps which preserve equality of distance. Studia Math. 45, 43-48 (1973)

34. Vroegindewey, P.G.: An algebraic generalization of a theorem of E.C.Zeeman. Indagationes Math. 36, 77-81 (1974)

35. Weyl,H.: Raum-Zeit Materie (1923), reprint: Berlin-Göttingen-Heidelberg: Springer 1961

36. Woodhouse, M. N.J.: The differentiability and causal structure of space time. J. Math. Phys. 14, 495-501 (1973)

37. Zeeman,E. C.: Causality implies the Lorentz-group. J. Math. Phys. 5, 490-498 (1964)

38. Zeeman, E. C.: The topology of Minkowski space. Topology 6, 161-170 (1967)

39. Knichal,V.: Die Bestimmung aller Transformationen, für welche die Null-Entfernung in den karthesischen Räumen mit indefiniter Metrik invariant bleibt. ATTI Sesto Congr. Mat. Ital. (11.-16.9.1959) 413-416

40. Pimenov, P. I.: Mat. Zametki 6, 361-369 (1969)

41. Alexandrov, A. D., Ovčinnikova, V. V.: Vestnik Leningrad. Univ. 8, 95-110 (1953)

42. Hawking, S.W., King, A.R., McCarthy, P.J.: A new topology for curved space-time which incorporates the causal, differential and conformal structures. J. Math. Phys., to appear

43. Göbel,R.: The smooth-path topology for curved space-time which incorporates the conformal structure and analytic Feynman tracks. J. Math. Phys., to appear

Communicated by J. Ehlers

Received October 13, 1974 
